\title{
Small-angle scattering and scale-dependent heterogeneity
}

\author{
Cedric J Gommes * \\ Department of Chemical Engineering, University of Liège B6A, 3 Allée du six août, \\ B-4000Liège, Belgium.E-mail: Cedric.Gommes@ulg.ac.be
}

\begin{abstract}
Although small-angle scattering is often discussed qualitatively in terms of material heterogeneity, when it comes to quantitative data analysis this notion becomes somehow hidden behind the concept of correlation function. In the present contribution, we define a quantitative measure of heterogeneity, we show how it can be calculated from scattering data, and we discuss its structural significance for the purpose of material characterisation.

Conceptually, the procedure consists in using a finite probe volume to define a local average density at any point of the material; the heterogeneity is then quantitatively defined as the fluctuations of the local average density when the probe volume is moved systematically through the sample. Experimentally, we show that the so-defined heterogeneity can be estimated by projecting the small-angle scattering intensity onto the form factor of the chosen probe volume. Chosing probe volumes of various sizes and shapes enables one to comprehensively characterise the heterogeneity of a material over all its relevant length-scales. We derive general results for asymptotically small and large probe in relation to the material surface area and integral range. We also
\end{abstract} PREPRINT: Journal of Applied Crystallography A Journal of the International Union of Crystallography 
show that the correlation function is equivalent to a heterogeneity calculated with a probe volume consisting of two points only.

The interest of scale-dependent heterogeneity for practical data analysis is illustrated with experimental SAXS patterns measured on a micro- and meso-porous material, on a gel, as well as on a semi-crystalline polyethylene sample. Using different types of probes to analyze a given scattering pattern enables one to focus on different structural characteristics of the material, which is particularly useful in the case of hierarchical structures.

\section{Introduction}

The general aim of small-angle scattering data analysis can be put as converting reciprocal-space data into real-space structural information. The underlying mathematics were developed by the founders of the field and they stand in a single sentence, namely: the scattered intensity is the Fourier transform of a density correlation function (Porod, 1948; Guinier \& Fournet, 1955; Debye et al., 1957). The relevant density differs according to the specific type of scattering - electron or scattering length densities for x-rays or neutrons, respectively - but the mathematics are the same. The apparent simplicity of that statement hides a wealth of difficult questions related notably to the numerical evaluation of correlation functions from scattering data (Glatter, 1977), the determination of non-trivial structural information (Tchoubar

\& Méring, 1969), the development and adjustment of structural models to scattering patterns (Pedersen, 1997; Svergun, 1999), the SAXS analysis of hierarchical structures (Gupta et al., 2006; Gommes et al., 2016), the enumeration of structures compatible with a given correlation function (Gommes et al., 2012a), etc. Any new, albeit mathematically equivalent, perspective on small-angle scattering may shed a new light on all these questions as well.

IUCr macros version 2.1.6: 2014/10/01 
The perspective we develop in the present paper is that of the material's heterogeneity. Although the word heterogeneous is often encountered in the context of small-angle scattering, it is generally used merely as a synonym for biphasic (or multiphasic). In that context, small-angle scattering is sometimes discussed as if it originated from the presence of interfaces (Ciccariello, 1988). An alternative and more general definition of heterogeneity is the one used in the field of geostatistics (Matheron, 1971; Chilès \& Delfiner, 1999). In that context, heterogeneity is a synonym for spatial variability.

Putting aside for a while the specific context of small-angle scattering, let us consider the general question of how to characterize quantitatively the heterogeneity of a structure. For the purpose of illustration, Figure 1 displays two structures having different heterogeneities. We shall refer to the white phase as the solid and the black phase as the pore, but the discussion is quite general. The two structures were designed to have identical volume fractions and specific surface areas (see Supporting Information), but they strongly differ by the spatial distribution of the solid. In the clipped Gaussian field model (Figure 1a), the solid regions are almost homogeneously distributed in space. By contrast, in the case of the Boolean model (Figure 1b) the solid forms clusters, which leads to pores with a broad size distribution. It is this type of difference that we wish to describe quantitatively, by defining a suitable measure of structural heterogeneity.

IUCr macros version 2.1.6: 2014/10/01 

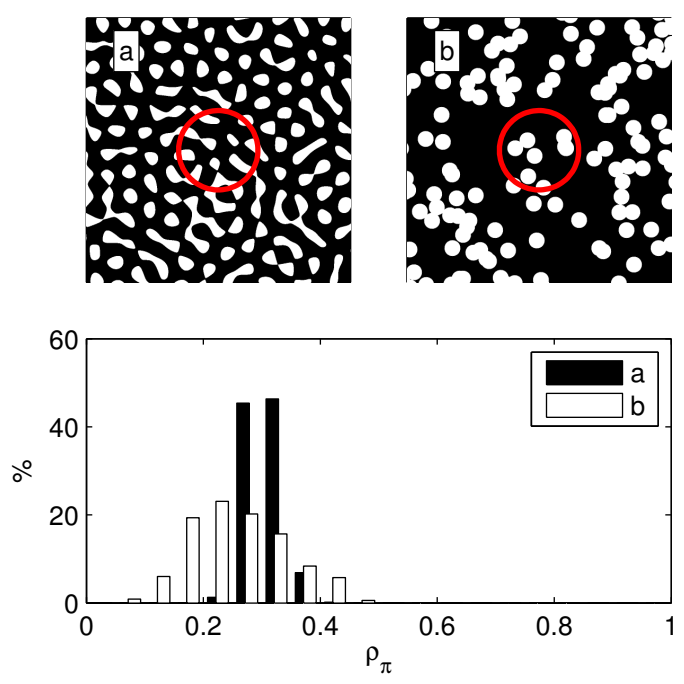

Fig. 1. Two qualitatively different structures ( $\mathrm{a}$ and $\mathrm{b}$ ) analysed with the same probe volume $\Pi$ (red disk). When the local average-density $\rho_{\pi}$ is calculated within the disk, the value fluctuates according to the actual position of the disk. The histogram (c) shows the values taken by $\rho_{\pi}$ when the disk is moved throughout the entire structure. We use the variance of the distributions of $\rho_{\pi}$ as a quantitative measure for the heterogeneity of the structure.

An equivalent way to look at the question of heterogeneity consists in assessing to what extent a local characteristic of the structure is representative of the whole. To be more specific consider a local probe volume $\Pi$ centred on a given point $\mathbf{x}$ of the material. In the case of Figure 1 the probe volume is the red disk. Averaging the density of the material inside the probe volume provides a local density, which depends on the particular position $\mathbf{x}$ of the probe; we refer to it as $\rho_{\pi}(\mathbf{x})$. For the structure in Figure 1a the local density is almost independent of the position $\mathbf{x}$ of the disk because the structure is homogeneous. On the contrary, for the structure in Figure 1b the disk may fall entirely inside a black region or be almost filled with white. This results in strong density fluctuations, which have to be interpreted as a very heterogeneous structure. This is confirmed by the histograms of $\rho_{\pi}$ shown in Figure 1 , which were obtained by moving the disk systematically over the entire structures. The average value of both distributions are identical and they coincide with the overall solid fraction (30\% 
for both structures a and b) but the variances are distinctly different. We define the heterogeneity of a structure as the variance $\sigma^{2}\{\Pi\}$ of the local average-density $\rho_{\pi}(\mathbf{x})$, calculated over all possible values of $\mathbf{x}$.

This definition of $\sigma^{2}\{\Pi\}$ enables one to characterise the heterogeneity of a material over a variety of length scales, via the use of probe volumes $\Pi$ with different sizes. This is illustrated in Figure 2 where the heterogeneity of a given structure is characterised with probe volumes consisting of disks having different radii. To keep the discussion general, we refer to the densities of the solid (white) and pore (black) as $\rho_{S}$ and $\rho_{P}$; their volume fractions are $\phi_{S}$ and $\phi_{P}=1-\phi_{S}$. If the disk is much larger than any characteristic size of the structure (Figure 2-b3), the values of the local average-density $\rho_{\pi}$ are distributed narrowly around the global average $\phi_{S} \rho_{S}+\phi_{P} \rho_{P}$. The narrowness of the distribution means that the structure is homogeneous on the scale of the disk. In the other extreme case where the disk is much smaller than any characteristic size of the structure, (Figure 2-b1) it generally falls entirely in one of the two phases. The local average-density $\rho_{\pi}$ then takes either the value $\rho_{S}$ or $\rho_{P}$ with probabilities $\phi_{S}$ and $\phi_{P}$, respectively. Because this corresponds to a binomial statistical distribution, the corresponding variance is

$$
\lim _{R \rightarrow 0} \sigma^{2}\{\Pi\}=\left(\rho_{S}-\rho_{P}\right)^{2} \phi_{S} \phi_{P}
$$

which coincides with the expression of Porod's invariant (Glatter \& Kratky, 1982; Sivia, 2011). In the particular case of Figure 2 with $\rho_{P}=0, \rho_{S}=1, \phi_{S}=0.3$ and $\phi_{P}=0.7$ the limiting value is $\sigma^{2}\{\Pi\} \simeq 0.21$. The variance takes smaller values for all finite disk sizes. The curve obtained by plotting the variance $\sigma^{2}\{\Pi\}$ as a function of the disk size (Figure 2c) provides a comprehensive characterisation of the structure heterogeneity over all length scales.

IUCr macros version 2.1.6: 2014/10/01 


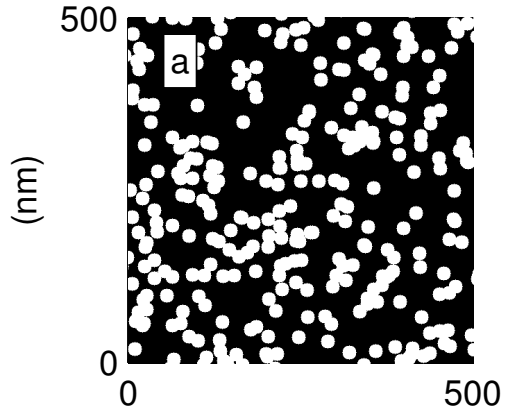

$(\mathrm{nm})$
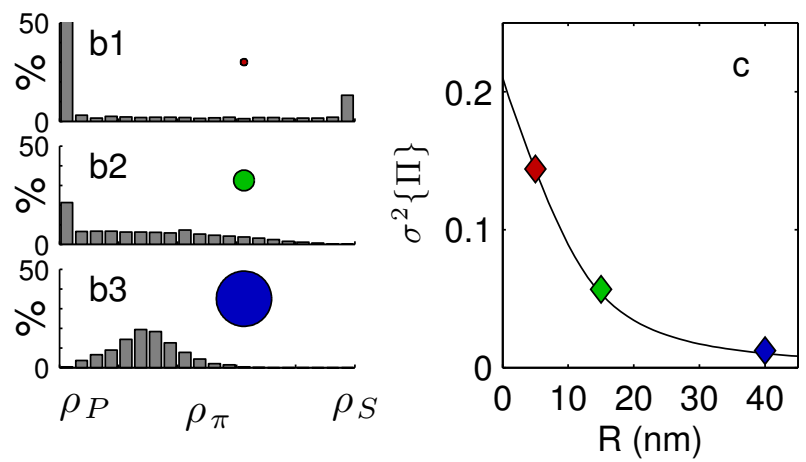

Fig. 2. When the procedure of Figure 1 is applied to a given structure (a) with probe volumes of increasing sizes (red, green, and blue disks with radii $R$ ), the corresponding distributions of the local average-density $\rho_{\pi}$ are size-dependent (b1 to b3). Considering the variance of the distributions as a function of the probe size (c) provides a comprehensive characterisation of the structure heterogeneity over all length scales. The three points in c were obtained numerically from the structure shown in a, and the solid line was obtained analytically from the known correlation function of the structure via Eq. (7). The main result of the paper is Eq. (14), which enables one to calculate the heterogeneity curve $\sigma^{2}\{\Pi\}$ starting from the scattering pattern.

The purpose of the present paper is twofold. On one hand, we show how scaledependent heterogeneity curves, similar to Figure 2c can be obtained experimentally from small-angle scattering patterns. On the second hand, we analyze the significance of these curves in term of the underlying structure. In a first section of the paper, we derive a mathematical relation between the variance of $\rho_{\pi}$ and the SAS intensity, which is valid for any type of probe volume. The simplest probe volumes we consider are spheres of various radii, which are the natural three-dimensional equivalent of the disks used in Figs. 1 and 2; we also discuss other types of probes that are easier to handle in reciprocal space. Afterwards, we build on Eq. (1) and explore further the structural significance of $\sigma^{2}\{\Pi\}$-versus-size for probes that are asymptotically small or large compared to the characteristic size of the structure. This enables us to devise new SAS data analysis procedures to measure surface areas, integral ranges, and to determine size distributions. Finally, in the discussion section, the methods are applied 
to experimental SAXS patterns measured on a micro- and mesoporous materials, on a gel, as well as on a semicrystalline polyethylene sample.

\section{Theoretical section}

\subsection{General relation between scale-dependent heterogeneity and scattering patterns}

In the introduction section, we have defined the local average density $\rho_{\pi}$ using a disk-shaped probe volume (see Figs. 1 and 2). Local averages can, however, be defined with any type of probe volumes and we write

$$
\rho_{\pi}(\mathbf{x})=\int \mathrm{d} V_{y} \Pi(\mathbf{y}-\mathbf{x}) \rho(\mathbf{y})
$$

where $\Pi($.$) can be any function that is integrable in three dimensions. Because the$ function $\Pi($.$) need not be defined on a finite support, we no longer refer to it as a probe$ volume in the rest of the paper but as a probe function, or simply as a probe. For the sake of convenience, the probes that we consider in the paper are all normalised to one, namely $\int \Pi(\mathbf{y}) \mathrm{d} V_{y}=1$. With this particular normalisation the definition of $\rho_{\pi}$ can be interpreted in terms of a low-pass filtering operation that is common to signal and image processing. By this process the filtered density map $\rho_{\pi}(\mathbf{x})$ is obtained by replacing the value at point $\mathbf{x}$ by the average value of $\rho$ in some neighbourhood centred on $\mathbf{x}$. In that sense, the passing from $\rho(\mathbf{x})$ to $\rho_{\pi}(\mathbf{x})$ is equivalent to a moving-average operation. An equation similar to Eq. (2) has also been introduced by Ruland (1971) to analyze the scattering from structures with interfaces having a finite thickness. For our present purpose, however, it has to be stressed that $\rho_{\pi}(\mathbf{x})$ does not correspond to any real density function. It is used here as a mathematical procedure to define the heterogeneity.

The particular case of a sphere with radius $R$ corresponds to the following probe

$$
\Pi_{R}(\mathbf{y})=\frac{3}{4 \pi R^{3}} \Theta(R-|\mathbf{y}|)
$$

IUCr macros version 2.1.6: 2014/10/01 
where $\Theta()$ is Heavyside's step function, which is equal to 1 if its argument is positive and to 0 otherwise. Another possibility consists in defining a Gaussian probe with variance $a$, namely

$$
\Pi_{a}(\mathbf{y})=\frac{1}{\left(2 \pi a^{2}\right)^{3 / 2}} \exp \left(\frac{-|\mathbf{y}|^{2}}{2 a^{2}}\right)
$$

With a Gaussian probe, points in the structure contribute less to the average value $\rho_{\pi}(\mathbf{x})$ if they are far away from $\mathbf{x}$, but there is no sharp cutoff distance. As we shall see later, this smooth transition in real space makes Gaussian probes easier to handle in reciprocal space. Throughout the rest of the paper we shall consider other types of probes. The present section is general and it applies to any of them.

The average value of $\rho_{\pi}(\mathbf{x})$ when $\mathbf{x}$ is uniformly distributed over the entire sample can be calculated directly from Eq. (2), yielding

$$
\left\langle\rho_{\pi}\right\rangle=\langle\rho\rangle
$$

where $\langle$.$\rangle denotes the average over all values of \mathbf{x}$. Throughout the paper, the brackets can also be interpreted as the expected value if the probe is positioned randomly in the structure. As discussed in the introduction section, the variance of $\rho_{\pi}(\mathbf{x})$ characterises the heterogeneity of the structure at the particular scale of the probe $\Pi$. It is defined as

$$
\sigma^{2}\{\Pi\}=\left\langle\rho_{\pi}^{2}\right\rangle-\left\langle\rho_{\pi}\right\rangle^{2}
$$

In this notation, the curled brackets $\{$.$\} highlight the fact that the variance has a$ functional dependence on the probe.

In order to relate the variance $\sigma^{2}\{\Pi\}$ to the properties of the probe and to $\rho(\mathbf{x})$, one may introduce the definition of $\rho_{\pi}$ through Eq. (2) into Eq. (6), and calculate explicitly the averages as integrals over $\mathbf{x}$. This leads to the following result

$$
\sigma^{2}\{\Pi\}=\int \chi_{\rho}(\mathbf{r}) \Omega_{\pi}(\mathbf{r}) \mathrm{d} V_{r}
$$

IUCr macros version 2.1.6: 2014/10/01 
where

$$
\chi_{\rho}(\mathbf{r})=\langle(\rho(\mathbf{x}+\mathbf{r})-\langle\rho\rangle)(\rho(\mathbf{x})-\langle\rho\rangle)\rangle
$$

is the correlation function of the density, and

$$
\Omega_{\pi}(\mathbf{r})=\int \Pi(\mathbf{x}+\mathbf{r}) \Pi(\mathbf{x}) \mathrm{d} V_{x}
$$

is the self-convolution of the probe. Equation (7) is a classical result of geostatistics (Serra, 1982; Chilès \& Delfiner, 1999). It seems to have been obtained first by Matheron (1971) and later rediscovered by Lu \& Torquato (1990) in the context of theoretical materials sciences. That relation has been applied in a wide variety of contexts, notably astronomy (Pietronero et al., 2002), materials engineering (Kanit et al., 2003), and statistical physics (Torquato \& Stillinger, 2003). In the present paper, we explore the significance and consequences of that general result in the particular field of smallangle scattering.

Because the only characteristic of $\rho(\mathbf{x})$ that enters Eq. (7) is the correlation function $\chi_{\rho}(\mathbf{r})$, it is possible to express $\sigma^{2}\{\Pi\}$ in terms of the scattered intensity alone. Before doing that, we shall first recall of few general results of elastic scattering theory (Sivia, 2011), if only to introduce some notations. The SAS intensity $I(\mathbf{q})$ is the Fourier transform of the correlation function $\chi_{\rho}(\mathbf{r})$, namely

$$
I(\mathbf{q})=\int \exp (i \mathbf{q} \cdot \mathbf{r}) \chi_{\rho}(\mathbf{r}) \mathrm{d} V_{r}
$$

where the relevant $\rho$ is either the electron density (SAXS) or the scattering length density (SANS). Calculating the inverse Fourier transform of this equation and evaluating it for $r=0$, one finds

$$
\chi_{\rho}(0)=\frac{1}{(2 \pi)^{3}} \int I(\mathbf{q}) \mathrm{d} V_{q}
$$

where $\mathrm{d} V_{q}$ is the volume element in reciprocal space, which converts to $4 \pi q^{2} \mathrm{~d} q$ in the case of isotropic scattering patterns. By the definition of $\chi_{\rho}$ in Eq. (8), the value IUCr macros version 2.1.6: 2014/10/01 
$\chi_{\rho}(0)$ is the variance of the density $\left\langle(\rho-\langle\rho\rangle)^{2}\right\rangle$. This quantity is generally referred to as $Q$, and it is given by Eq. (1) in the particular case of a two-phase structure. In the context of scattering studies, it is common to normalise the correlation function by $Q$ and introduce Debye's correlation function $\gamma(\mathbf{r})$ as

$$
\gamma(\mathbf{r})=\chi_{\rho}(\mathbf{r}) / Q
$$

which satisfies $\gamma(0)=1$.

The relation between the heterogeneity $\sigma^{2}\{\Pi\}$ and the SAS intensity $I(\mathbf{q})$ for any probe is obtained by expressing $\chi_{\rho}(\mathbf{r})$ in Eq. (7) as the inverse Fourier transform of $I(\mathbf{q})$. This leads to

$$
\sigma^{2}\{\Pi\}=\frac{1}{(2 \pi)^{3}} \int I(\mathbf{q}) P_{\pi}(\mathbf{q}) \mathrm{d} V_{q}
$$

where $P_{\pi}(\mathbf{q})$ is the Fourier transform of $\Omega_{\pi}(\mathbf{r})$. In general, powder scattering patterns are measured experimentally, in which case $I(\mathbf{q})$ depends only on the modulus of the scattering wave vector $q=|\mathbf{q}|$. In this case the relation becomes

$$
\sigma^{2}\{\Pi\}=\frac{1}{(2 \pi)^{3}} \int_{0}^{\infty} I(q) P_{\pi}(q) 4 \pi q^{2} \mathrm{~d} q
$$

where $P_{\pi}(q)$ is the orientation-average of $P_{\pi}(\mathbf{q})$, which can be thought of as the form factor of the probe П. Equation (14) is central in the present paper. It shows how the heterogeneity curves $\sigma^{2}\{\Pi\}$, equivalent to the right panel of Figure 2, can be calculated from experimental small-angle scattering data, for any type of probe. In order to calculate the heterogeneity of a structure as defined in Figure 1, one has to multiply the experimental scattering pattern by the form factor of the chosen probe and to integrate over the entire reciprocal space. For further purposes, the real-space structure and the form factors of the spherical and Gaussian probes are shown in Figure $3 \mathrm{a}$ and $3 \mathrm{~b}$. The analytical expressions are given in Table 1.

IUCr macros version 2.1.6: 2014/10/01 


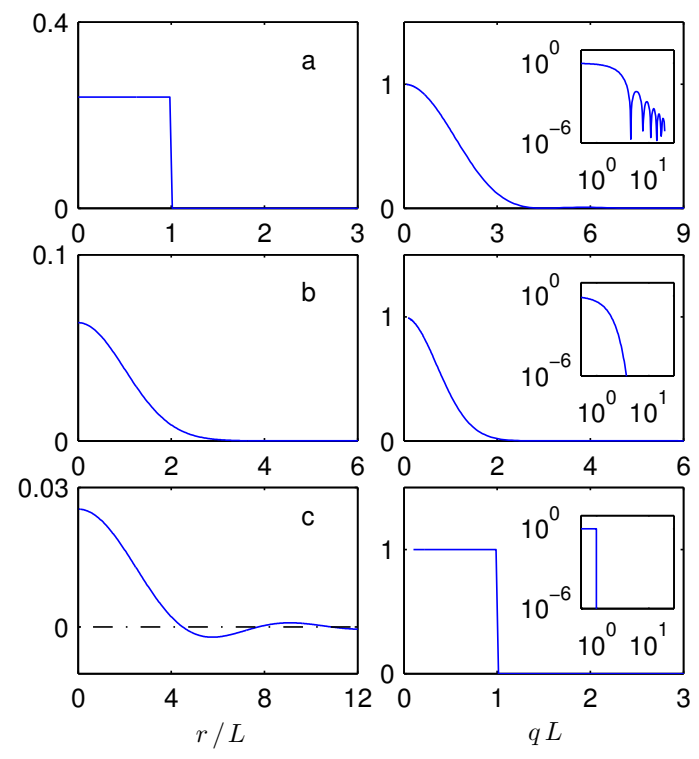

Fig. 3. Three probe functions $\Pi$ considered in the present work (left, real space), together with their form factors $P_{\pi}(q)$ (right, reciprocal space). The insets are the same plots on double logarithmic scales. From top to bottom: the sphere (a), the Gaussian (b), and the $q$-sphere (c). See Table 1 for the properties of these probes. The size $L$ of the probe is the radius for the sphere, the standard deviation for the Gaussian, and the inverse cutoff frequency for the $q$-sphere.

The central result of the paper, Eq (14) can also be obtained on the following more intuitive grounds, by following the approach used by Ruland (1971) to analyse the scattering by systems with interfaces having a finite thickness. Because a multiplication in reciprocal space converts to a convolution in real space, the product $I(q) \times P_{\pi}(q)$ can be thought of as the SAS pattern corresponding to the filtered density $(\rho * \Pi)(\mathbf{x})$, where $*$ is a convolution product. The latter convolution is nothing but $\rho_{\pi}(\mathbf{x})$ defined in Eq. (2) as a moving-average filter. Equation (14) then follows directly from Eq. (11) applied to the SAS of the filtered density distribution, or equivalently from Parseval's theorem. 


\begin{tabular}{l|ccc} 
& Sphere & Gaussian & $q$-Sphere \\
\hline$\Pi(\mathbf{y})$ & Eq. $(3)$ & Eq. $(4)$ & Eq. $(27)$ \\
$P_{\pi}(q)$ & $9[\sin (q R)-q R \cos (q R)]^{2} /(q R)^{6}$ & $\exp \left(-q^{2} a^{2}\right)$ & $\Theta(\nu-q)$ \\
Size $L$ & Radius $R$ & Standard deviation $a$ & Inverse cutoff $1 / \nu$ \\
\hline$\alpha_{\pi}^{(0)}$ & 1 & 1 & 1 \\
$\alpha_{\pi}^{(1)}$ & $35 / 36$ & $4 / \sqrt{\pi}$ & $4 / \pi$ \\
$\alpha_{\pi}^{(2)}$ & $6 / 5$ & 6 & 0 \\
$\alpha_{\pi}^{(3)}$ & $32 / 21$ & $32 / \sqrt{\pi}$ & $-16 / \pi$ \\
\hline$\beta_{\pi}^{(0)}$ & $3 /(4 \pi)$ & $1 /\left(8 \pi^{3 / 2}\right)$ & $1 /\left(6 \pi^{2}\right)$ \\
$\beta_{\pi}^{(1)}$ & $-9 /(16 \pi)$ & 0 & 0 \\
$\beta_{\pi}^{(2)}$ & 0 & $-1 /\left(16 \pi^{3 / 2}\right)$ & $-1 /\left(30 \pi^{2}\right)$ \\
$\beta_{\pi}^{(3)}$ & $9 /(32 \pi)$ & 0 & 0
\end{tabular}

Table 1. Characteristics of a the main probes used in the text, with notably their real-space structure $\Pi(\mathbf{y})$ and their form factor $P_{\pi}(q)$. The constants $\alpha_{\pi}^{(n)}$ and $\beta_{\pi}^{(n)}$ are defined by Eqs. (23) and (32). These values are required to determine the surface area and the integral range from the slopes of the relevant scale-dependent heterogeneity curves.

\subsection{Scale-dependent heterogeneity as a generalised correlation function}

It order to put the concept of heterogeneity $\sigma^{2}\{\Pi\}$ in a broader perspective, it is interesting to consider the particular probe consisting in only two points at a distance $\mathbf{r}$ from one another.This can be formalised as

$$
\Pi_{2 r}(\mathbf{y})=\frac{1}{2}\left[\delta\left(\mathbf{y}-\frac{\mathbf{r}}{2}\right)+\delta\left(\mathbf{y}+\frac{\mathbf{r}}{2}\right)\right]
$$

where $\delta()$ is Dirac's function.

In order to keep the discussion simple, we shall restrict the analysis of the present section to the same two-phase system as in the introduction, comprising a solid phase $S$ and pore space $P$, having densities $\rho_{S}$ and $\rho_{P}$, and volume fractions $\phi_{S}$ and $\phi_{P}$. In that case the variance of $\rho(\mathbf{x})$ is given by the classical form of Porod's invariant $Q=\left(\rho_{S}-\rho_{P}\right)^{2} \phi_{S} \phi_{P}$ as in Eq. (1).

The second-order spatial statistics of biphasic systems are conveniently described by the so-called stick probability functions $P_{S S}(r), P_{P P}(r), P_{S P}(r)$, which are equal to the probabilities that pairs of points at distance $r$ from one another belong both to phase $S$, both to phase $P$, or one to each phase (Goodisman \& Brumberger, 1971; Ciccariello IUCr macros version 2.1.6: 2014/10/01 
et al., 1981; Torquato, 2000). The latter probabilities are related to the correlation function $\gamma(r)$ by

$$
\begin{aligned}
P_{S S}(r) & =\phi_{S}\left[\phi_{P} \gamma(r)+\phi_{S}\right] \\
P_{P P}(r) & =\phi_{P}\left[\phi_{S} \gamma(r)+\phi_{P}\right] \\
P_{S P}(r) & =\phi_{S} \phi_{P}[1-\gamma(r)]
\end{aligned}
$$

These relations can be obtained by noting that $P_{S S}(r)+P_{S P}(r)=\phi_{S}$ for any value of $r$, and that a similar relation holds when inverting phases $S$ and $P$.

With these notations in mind, the values taken by $\rho_{\pi}$ for the two-point probe $\Pi_{2 r}$ are the following

$$
\rho_{\pi}=\left\{\begin{array}{lll}
\rho_{S} & \text { with probability } & P_{S S}(r) \\
\left(\rho_{S}+\rho_{P}\right) / 2 & \text { with probability } & 2 \times P_{S P}(r) \\
\rho_{P} & \text { with probability } & P_{P P}(r)
\end{array}\right.
$$

The factor 2 in second case accounts for the fact that $P_{S P}(r)=P_{P S}(r)$. Using these probabilities, the heterogeneity $\sigma^{2}\{\Pi\}$ can be calculated explicitly as $\left\langle\rho_{\pi}^{2}\right\rangle-\left\langle\rho_{\pi}\right\rangle^{2}$, which eventually leads to

$$
\sigma^{2}\{\Pi\}=Q \frac{1+\gamma(\mathbf{r})}{2}
$$

This equation shows that the correlation function and the heterogeneity are linearly related to one another. In other words, the two functions carry the same structural information.

The meaning of Eq. (18) is conveniently analysed from a statistical perspective. Imagine that we want to estimate the true global density $\langle\rho\rangle$ of the material, and that we do it by picking up a single point randomly in the structure. The error on that single measurement would be extremely large, because the estimated density would be either $\rho_{S}$ or $\rho_{P}$. The variance of that one-point estimation is exactly $Q$. A better estimation of $\langle\rho\rangle$ consists in taking two random points and calculating their average value for the purpose of reducing the variance. The variance of this two-point estimation depends 
on the distance $r$ between the two points, as predicted by Eq. (18). If the two points are very close to each other, the variance is not reduced because the two points are likely to belong to the same phase $(\gamma \simeq 1)$. On the contrary, the variance is the lowest if the points are far apart because this minimises their correlation $(\gamma \simeq 0)$, so that the variance is reduced by a factor of two. It is therefore natural that the heterogeneity with two-point probes be directly related to the correlation function $\gamma(r)$. The same type of reasoning applies to the three-point probes, consisting of three Dirac's measures put at the vertices of an equilateral triangle with side $r$. We show in the Supporting Information that the heterogeneity is $\sigma^{2}\{\Pi\}=Q(1+2 \gamma(r)) / 3$ for that particular probe.

The reason why $\gamma(r)$ appears in such a simple form in Eq. (18) as well as for the three-point probe, is that the only distance that exists between all the points that make up the probe is $r$. In the case of a sphere with radius $R$, all distances from $r=0$ to $r=2 R$ are possible between pairs of points in the probe. In that case, it is natural for the heterogeneity to be a linear combination of the values of $\gamma(r)$ evaluated at all possible distances, from 0 to $2 R$. This is the meaning of Eq. (7), because the self-correlation $\Omega_{\pi}(r)$ can be thought of in terms of a pair-distribution function of the probe $\Pi$.

For the purpose of analysing the relation of the two-point probe $\Pi_{2 r}(\mathbf{y})$ with smallangle scattering via Eq. (14), it is also interesting to calculate its form-factor. It is calculated as the rotationally-averaged square modulus of the Fourier transform of Eq. (15), which leads to

$$
P_{\pi}(q)=\frac{1}{2}\left(1+\frac{\sin (q r)}{q r}\right)
$$

With that particular expression, Eq. (14) reduces to the classical form of an inverse Fourier transform. It has to be stressed, however, that Eq. (14) is more general in that it applies to any type of probe. From that perspective the correlation function $\gamma(r)$, IUCr macros version 2.1.6: 2014/10/01 
as it appears in Eq. (18) is just one among many other types of heterogeneities that are related to the SAS intensity $I(q)$. Any type of probe can in principle be used to generate a heterogeneity curve $\sigma^{2}\{\Pi\}$ versus size, which carries the same structural information as $\gamma(r)$, only expressed differently.

\section{Structural significance of the heterogeneity}

The structural significance of the heterogeneity $\sigma^{2}\{\Pi\}$ is conveniently discussed based on a well-defined structure. For that purpose, the present section is illustrated with the hierarchical model of porous material shown in the inset of Figure 4. That model comprises both micro- and meso-pores. On the mesopore-scale, the structure has pores with approximate size $100 \AA$, which fill $50 \%$ of the volume. The remainder volume is occupied by a solid that is itself microporous, i.e. with pores smaller than $20 \AA$. The latter micropores fill $30 \%$ of space. This results in a material with an overall porosity of $65 \%$. Based on the parameters of the model the total surface area is $364 \mathrm{~m}^{2} / \mathrm{cm}^{3}$, of which $132 \mathrm{~m}^{2} / \mathrm{cm}^{3}$ are due to the mesopores and the remainder to the micropores. The mathematical details are available in the Supporting Information.

IUCr macros version 2.1.6: 2014/10/01 


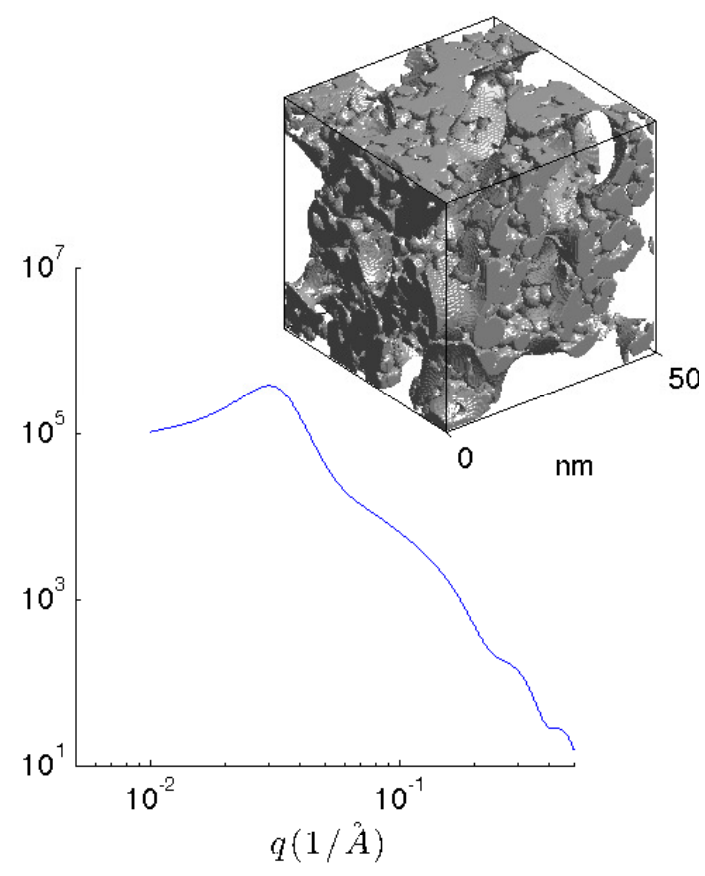

Fig. 4. SAS pattern $I(q)$ of the two-scale model of porous material used to illustrate the theoretical aspects of the paper. The structure comprises both mesopores and micropores (see Supporting Information for details). A particular realisation is shown in the inset.

As we discussed when introducing Eq. (2) the quantity $\rho_{\pi}(\mathbf{x})$ can be interpreted as a low-pass filtered version of $\rho(\mathbf{x})$, where the density at each point of the material is replaced by the average value calculated in a neighbourhood defined by the probe $\Pi(\mathbf{y})$. This is illustrated in Figure 5 with a 2D realisation of the same model as in Figure 4, in which the grey level stands for the density. The density map of the material $\rho(\mathbf{x})$ is biphasic and comprises only the values 0 and 1 with sharp boundaries. When the size of the probe $\Pi$ is progressively increased, the filtered density $\rho_{\pi}(\mathbf{x})$ progressively looses all its features starting from the smallest: first the boundaries are blurred, then the smallest objects are smoothed out, and all the structure of $\rho(\mathbf{x})$ is eventually filtered out for very large probes. The scale-dependent heterogeneity curve $\sigma^{2}\{\Pi\}$-versus-size, as shown in Figure 2c is an objective measure of what is left of the structure after applying a filter of a given size.

IUCr macros version 2.1.6: 2014/10/01 


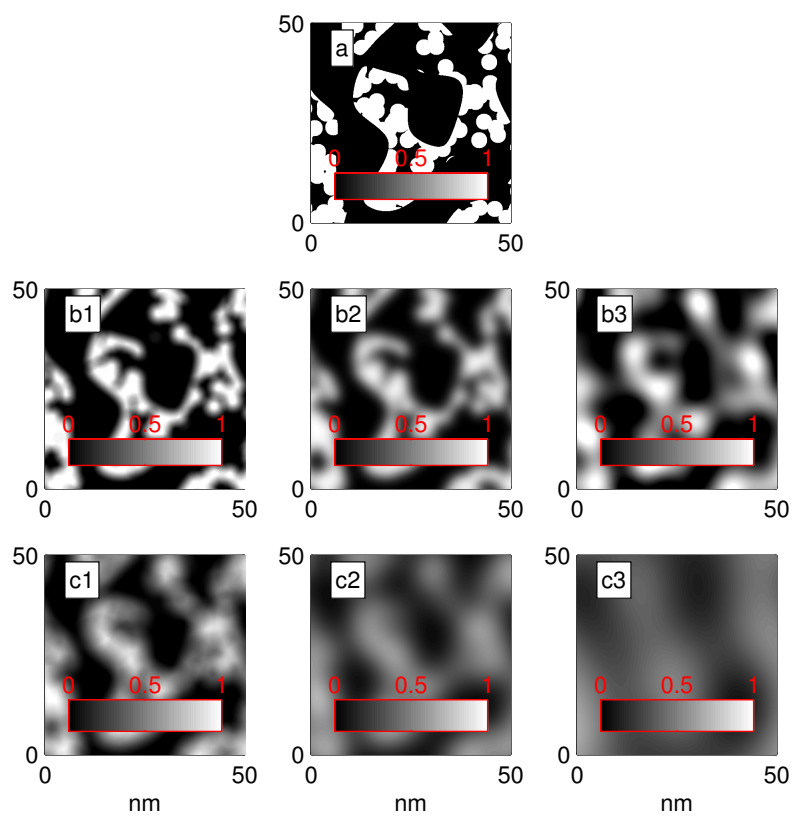

Fig. 5. Example of a biphasic structure (a) corresponding to a $2 \mathrm{D}$ realisation of the model in Figure 4, and the resulting $\rho_{\pi}(\mathbf{x})$ obtained with disk-like (b1-c1), Gaussian (b2-c2) and $q$-spherical (b3-c3) probes. The size of the probe is $L=1.5 \mathrm{~nm}$ (b1-b3) and $L=3.5 \mathrm{~nm}$ (c1-c3). See Table 1 for the exact meaning of the size $L$ for the three different windows.

It is a common procedure in image analysis, and particularly in mathematical morphology, to characterise a structure by analysing how it is modified further to the application of a given filter (Soille, 1999). The measurement of size distributions via opening granulometry typically follows that general scheme (Serra, 1982). In the context of image analysis the most efficient filters are non-linear and one generally measures the volume of objects. If one wants to apply the same type of procedure to SAS data analysis, one can only apply linear filters because the structural data is available in the reciprocal space. Moreover, the only measurement that one can make on the filtered structure is the variance $\sigma^{2}\{\Pi\}$ via Eq. (14). Although these constraints considerably limit the potential of the method, they are inherent to scattering methods. Moreover, they do not preclude the measurement of important structural characteristic of the material, as we now discuss. 


\subsection{Asymptotically small probes}

We start our discussion of the structural significance of $\sigma^{2}\{\Pi\}$ in the case where the probe is smaller than the characteristic size of the structure. For that purpose, consider again a biphasic structure with pore and solid phases $P$ and $S$ and interface area $A_{P S}$, analysed with a spherical probe with radius $R$. If the radius $R$ is much smaller than the radii of curvature of the interface, the density maps $\rho(\mathbf{x})$ and $\rho_{\pi}(\mathbf{x})$ are different only in a small layer of thickness $2 R$ on both sides of the interface. The effect of the filtering is to replace the density in that layer, which was initially either $\rho_{S}$ or $\rho_{P}$, by values that are evenly distributed between $\rho_{P}$ and $\rho_{S}$. The histogram in Figure 2-b1 is representative of that situation. Because the volume fraction of the layer that is affected by the filtering is proportional to $A_{P S} \times R$, one expects that the decrease of $\sigma^{2}\{\Pi\}$ with $R$ should be proportional to the interface area of the material.

This analysis can be made quantitative by particularising the general expression of $\sigma^{2}\{\Pi\}$ in Eq. (7) to the case of small probes. Assuming a probe much smaller than the characteristic size of the structure is equivalent to assuming that the function $\Omega_{\pi}(r)$ decreases to 0 much faster than $\chi_{\rho}(r)$, or than $\gamma(r)=\chi_{\rho}(r) / Q$. In Eq. (7), the correlation function $\gamma(r)$ can therefore be approximated as a limited Taylor development because all higher-order terms are significant only for values of $r$ where $\Omega_{\pi}(r)$ is vanishingly small. The approximation is

$$
\gamma(r) \simeq 1+\gamma^{(1)} r+\frac{1}{2} \gamma^{(2)} r^{2}+O\left(r^{3}\right)
$$

where $\gamma^{(n)}$ is the $n^{\text {th }}$ derivative of $\gamma(r)$ evaluated at $r=0$. Using this expression, the general relation between $\sigma^{2}\{\Pi\}$ and $\gamma(r)$ becomes

$$
\frac{\sigma^{2}\{\Pi\}}{Q}=\mu_{0}^{\Omega}+\gamma^{(1)} \mu_{1}^{\Omega}+\frac{1}{2} \gamma^{(2)} \mu_{2}^{\Omega}+\ldots
$$

where we have used the notation $\mu_{n}^{\Omega}$ for the moments of $\Omega_{\pi}(r)$, i.e.

$$
\mu_{n}^{\Omega}=\int_{0}^{\infty} r^{n} \Omega_{\pi}(r) 4 \pi r^{2} \mathrm{~d} r
$$

IUCr macros version 2.1.6: 2014/10/01 
Quite generally, the moment of order $n$ is proportional to the $n^{\text {th }}$ power of the probe size $L$. It is therefore convenient to introduce the proportionality constant as

$$
\mu_{n}^{\Omega}=\alpha_{\pi}^{(n)} L^{n}
$$

In the case of the spherical probe we chose $L$ as the radius, and for the Gaussian probe we identify $L$ with the standard deviation. The corresponding values of $\alpha_{\pi}^{(n)}$ for the spherical and Gaussian probes are calculated in the Supporting Information, and they are given in Table 1 for $n=0$ to 3 . Because of the normalisation $\int \Pi(\mathbf{y}) \mathrm{d} V_{y}=1$, all the probes of the present paper satisfy $\alpha_{\pi}^{(0)}=1$.

The derivatives of the correlation function for small values of $r, \gamma^{(n)}$, carry structural information about the interfaces of the material. For any system comprising two phases $P$ and $S$, the first derivative $\gamma^{(1)}$ is proportional to the interface area $A_{P S}$ (Debye et al., 1957), namely

$$
\gamma(r) \simeq 1-\frac{1}{4} \frac{A_{P S}}{\phi_{P} \phi_{S}} r+\ldots
$$

and the following terms depend on the smoothness and curvature of the interface (Kirste \& Porod, 1962; Ciccariello, 1995). We shall be mainly interested here in the linear term in $\gamma(r)$, which leads to the following linear relation between the scaledependent heterogeneity and the probe size

$$
\frac{\sigma^{2}\{\Pi\}}{Q} \simeq 1-\frac{\alpha_{\pi}^{(1)}}{4} \frac{A_{P S}}{\phi_{P} \phi_{S}} L+\ldots
$$

which enables one to estimate the specific surface area from the initial slope of the heterogeneity curves. Although different heterogeneity curves are obtained with different types of probes, the different values of $\alpha_{\pi}$ given in Table 1 enable one to obtain the same surface area $A_{P S}$ independently of the probe. For the purpose of data analysis, one can therefore select the type of probe that minimises the impact of experimental uncertainties. In particular, we now discuss how the estimation of surface area can be made insensitive to scattering data extrapolation by using an appropriate probe. 
Figures $6 \mathrm{a}$ and $6 \mathrm{~b}$ display the size-dependent heterogeneity $\sigma^{2}\{\Pi\}$ calculated from the SAXS pattern in Figure 4 with the spherical and Gaussian probes. The solid black line corresponds to Eq. (25) with the known surface area $A_{P S}$ of the model, and the value of $\alpha_{\pi}^{(1)}$ corresponding to the probe. A practical difficulty when calculating $\sigma^{2}\{\Pi\}$ from the SAS patterns via Eq. (14) is the evaluation of the integral, which requires extrapolating the data outside of the experimentally measured $q$-range. This is illustrated by the insets of the figure: when no extrapolation is used (black symbols) the heterogeneity $\sigma^{2}\{\Pi\}$ behaves quadratically close to the origin so that it is impossible estimate the specific surface area. On the other hand, when the scattering data $I(q)$ is extrapolated with a $q^{-4}$ Porod law before evaluating the integral, the values of $\sigma^{2}\{\Pi\}$ are found to be in fair agreement with Eq. (25).

IUCr macros version 2.1.6: 2014/10/01 

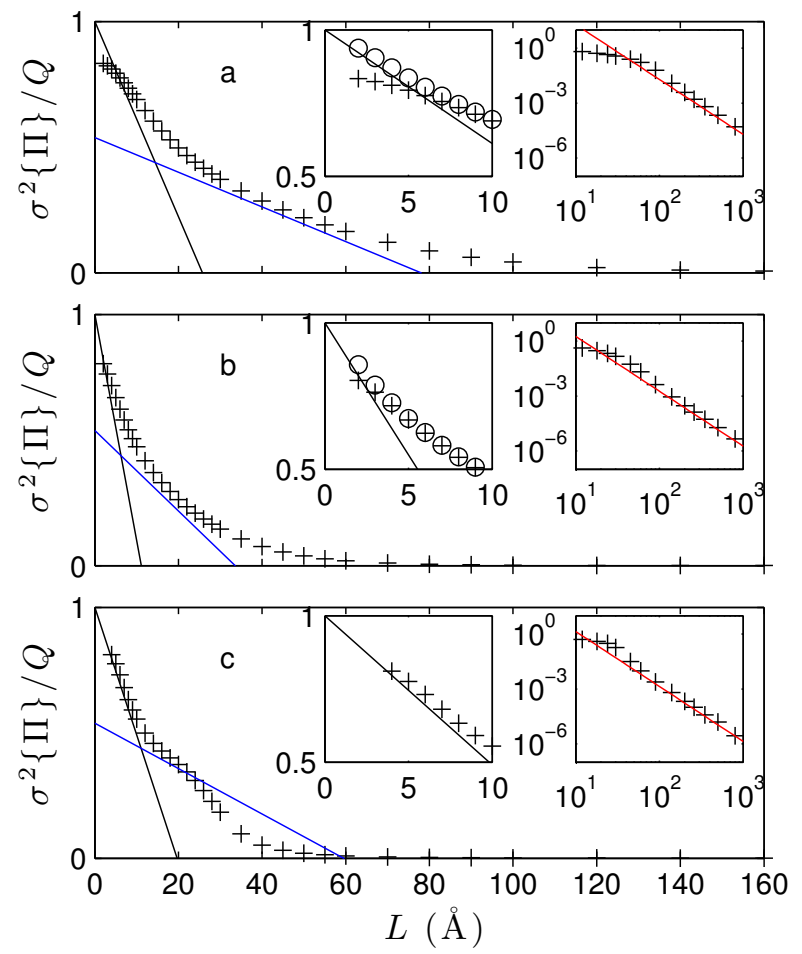

Fig. 6. Scale-dependent heterogeneity curves $\sigma^{2}\{\Pi\}$ calculated from the SAXS data in Figure 4 using Eq. (14) for spherical (a), Gaussian (b) and $q$-spherical (c) probes. The insets display a magnified view of the origin (left) and the same data on double logarithm scales (right). In the first case, the circles are obtained after extrapolating the data by a Porod law to calculate $\sigma^{2}\{\Pi\}$. The solid black line is the linear smallprobe approximation Eq. (25), the blue line is Eq. (39), and the red line is the large-probe approximation Eq. (33).

The extrapolation of $I(q)$ is a customary procedure when analysing experimental data, e.g. for the evaluation of the total scattered intensity $Q$. However, it is always open to the criticism that one is forcing the data into a Porod law, which might not actually be present. For evaluating the specific surface area via the slope of $\sigma^{2}\{\Pi\}$, the sensitivity to extrapolation can be reduced by using a probe that is more compact in reciprocal space than the sphere. This is notably the case for the Gaussian, which leads to values of $\sigma^{2}\{\Pi\}$ that are not very dependent on the data extrapolation (see inset of Figure 6b). The extrapolation can be altogether avoided by using a probe that 
is compact is reciprocal space. The simplest such probe has the following form factor

$$
P_{J}(q)=\Theta(\nu-q)
$$

where $\Theta()$ is Heavyside's step function. Because this equation describes a sphere in reciprocal space with radius $q=\nu$, we refer to this probe as a $q$-sphere. The filtering operation corresponding to this probe (i.e. for generating the corresponding density map $\left.\rho_{\pi}(\mathbf{x})\right)$ is a perfect low-pass filtering of the structure with all spatial frequencies larger than $\nu$ removed. Because the probe is compact in reciprocal space it is necessarily extended in real space. The real-space structure is obtained via a Fourier transform of Eq. (26), which leads to

$$
J_{\nu}(\mathbf{y})=\frac{3 \nu^{3}}{2 \pi^{2}} \frac{\sin (\nu|\mathbf{y}|)-(\nu|\mathbf{y}|) \cos (\nu|\mathbf{y}|)}{(\nu|\mathbf{y}|)^{3}}
$$

Under that form, it is apparent that the cutoff frequency $\nu$ is related to the real-space size of the probe via the inverse relation $L=1 / \nu$. The real- and reciprocal-space structures of the probe are shown in Figure 3c.

The scale-dependent heterogeneity of the model in Figure 4 based on $q$-spheres is shown in Figure 6c. It exhibits a sharp linear portion for small values of $L$, the slope of which nicely matches Eq. (25). The sharpness of the linear regions is desirable from the point of view of data analysis because it makes the slope estimation more accurate. Mathematically, the sharpness results from the fact that the second moments $\mu_{2}^{\Omega}$ is equal to zero for the $q$-sphere (see the values of $\alpha_{\pi}^{(2)}$ in Table 1 ). The quadratic term in Eq. (21) is therefore always equal to zero for a $q$-sphere, which increases the linearity of $\sigma\{\Pi\}$. Finally, we note that in addition to not requiring any scattering data extrapolation, the practical estimation of $\sigma^{2}\{\Pi\}$ from SAXS data $I(q)$ is particularly simple in the case of $q$-spheres. Indeed, Eq. (14) reduces to

$$
\sigma^{2}\{\Pi\}(\nu=q)=\frac{1}{2 \pi^{2}} \int_{0}^{q} I(q) q^{2} \mathrm{~d} r
$$

IUCr macros version 2.1.6: 2014/10/01 
which can easily be calculated from an experimental scattering pattern. Therefore, from the point of view of surface area determination, $q$-sphere is clearly more convenient a probe than both the sphere and the Gaussian.

The model in Figure 4 was designed to have a hierarchical structure displaying two families of pores, micro- and meso-pores, which are also visible in the $2 \mathrm{D}$ realisation in Figure 5a. It is interesting to see whether that feature is apparent in the heterogeneity curves $\sigma^{2}\{\Pi\}$. It seems that the $q$-spherical probe is more efficient than the sphere and Gaussian in that respect too. A second linear portion at intermediate values of $L$ is clearly visible in Figure 6c, which is unclear in Figs 6a and 6b. We shall come back to the quantitative analysis of that second linear region in the section devoted to the estimation of size distributions.

\subsection{Asymptotically large probes}

We have shown in the previous section how the small-probe behaviour of $\sigma^{2}\{\Pi\}$ enables one to determine the specific surface area from scattering data. We now turn to the structural information that can be obtained with probes that are much larger than the characteristic size of the structure. This is the typical situation sketched in Figure 2 b3. As we shall see, the relevant information in the heterogeneity curve is the so-called integral range of the structure. This is a central characteristic in the field of geostatistics (Lantuejoul, 1991) but it is seldom discussed in the context of scattering studies.

In the case of large probes the relation between the scale-dependent heterogeneity $\sigma^{2}\{\Pi\}$ and the correlation function can be simplified by approximating the integral in Eq. (7) via a limited Taylor development for the probe self-correlation $\Omega_{\pi}(r)$. This is justified here because $\gamma(r)$ decreases faster than $\Omega_{\pi}(r)$, so that higher-order terms in $\Omega_{\pi}(r)$ are significant only when $\gamma(r)$ is vanishingly small. The Taylor development

IUCr macros version 2.1.6: 2014/10/01 
leads to following series

$$
\frac{\sigma^{2}\{\Pi\}}{Q}=\Omega^{(0)} \mu_{0}^{\gamma}+\Omega^{(1)} \mu_{1}^{\gamma}+\frac{1}{2} \Omega^{(2)} \mu_{2}^{\gamma}+\ldots
$$

Compared to the case of small probes Eq. (21), the roles of the moments and of the derivatives are now inverted: the relevant moments are those of the correlation function

$$
\mu_{n}^{\gamma}=\int_{0}^{\infty} r^{n} \gamma(r) 4 \pi r^{2} \mathrm{~d} r
$$

and the derivatives are those of the probe's self-correlation

$$
\Omega^{(n)}=\left[\frac{\mathrm{d}^{n} \Omega_{\pi}(r)}{\mathrm{d} r^{n}}\right]_{r \rightarrow 0}
$$

Because the normalisation of the probes are equivalent to $\mu_{0}^{\Omega}=1$, the $\mathrm{n}^{\text {th }}$ derivative $\Omega^{(n)}$ is proportional to the power $L^{-(3+n)}$. In particular, the moment of order $0 \Omega_{\pi}(r=$ 0 ) is inversely proportional to the volume of the probe, i.e. to $L^{-3}$. We define the proportionality constant $\beta_{\pi}^{(n)}$ as follows

$$
\Omega^{(n)}=\beta_{\pi}^{(n)} / L^{3+n}
$$

The values of $\beta_{\pi}^{(n)}$ are calculated in the Supporting Information and they are reported in Table 1 for the main probes discussed in the paper. Using this notation, the scaledependent heterogeneity behaves as

$$
\frac{\sigma^{2}\{\Pi\}}{Q}=\mu_{0}^{\gamma} \frac{\beta_{\pi}^{(0)}}{L^{3}}+\mu_{1}^{\gamma} \frac{\beta_{\pi}^{(1)}}{L^{4}}+\ldots
$$

in the asymptotic limit of large probes. Equation (33) describes how the variances of the distributions shown in Figure 2 decreases when larger probes are used. From the point of view of data analysis, this shows how the scale-dependent heterogeneity can be used to estimate the moments of the correlation function.

The $1 / L^{3}$ decrease of $\sigma^{2}\{\Pi\}$ predicted from Eq. (33) is apparent when the scaledependent heterogeneity is plotted on double logarithmic scales (see the insets of IUCr macros version 2.1.6: 2014/10/01 
Figure 6). The leading term is proportional to $\mu_{0}^{\gamma}$, i.e. to the integral of the correlation function $\gamma(r)$. In the field of geostatistics, this is a quantity that is referred to as the integral range of the structure (Lantuejoul, 1991). Because it is not a usual concept in the context of scattering studies, we shall discuss its meaning is some detail. In the context of statistical physics and assuming thermal equilibrium, this integral is related to the compressibility of the system (Chandler, 1987). That quantity, however, has a universal geometrical meaning that is independent of any assumption on the system. Dimensionally, the integral range $\mu_{0}^{\gamma}$ is a volume. To understand its structural significance consider the case of a spherical probe, for which $L^{3} / \beta_{\pi}^{(0)}$ is equal to the probe volume $V_{\pi}=4 \pi R^{3} / 3$. With that in mind, the leading term in Eq. (33) can be written as

$$
\sigma^{2}\{\Pi\} \simeq \frac{Q}{N}
$$

with $N=V_{\pi} / \mu_{0}^{\gamma}$. This equation is best understood in the same spirit as our discussion of Eq. (18), namely by considering $\sigma^{2}\{\Pi\}$ as the variance of a density estimation. In that context $Q$ is the variance associated with a one-point estimation of the material density. From the perspective variance reduction through averaging, Eq. (34) means that the entire volume of the probe counts as much as $N$ individual and uncorrelated point-wise measurements of the density. In other words, from a statistical point of view every volume $\mu_{0}^{\gamma}$ of the material counts as much as one point only. In that sense the integral range is the natural scale of the structure (Lantuejoul, 1991). Typically, if one is interested in a representative volume element of a structure, it has to be many times larger than $\mu_{0}^{\gamma}$ (Kanit et al., 2003). The integral range is obtained from scattering pattern as

$$
\mu_{0}^{\gamma}=\lim _{q \rightarrow 0} \frac{I(q)}{Q}
$$

which results from estimating Eq. (10) for $q=0$.

Interestingly, the integral range $\mu_{0}^{\gamma}$ need not always take finite values. For example, IUCr macros version 2.1.6: 2014/10/01 
some fractal structures have a correlation function $\gamma(r)$ that decreases more slowly than $1 / r^{3}$, which leads to infinite values for $\mu_{0}^{\gamma}$. Another interesting case is when $\mu_{0}^{\gamma}$ becomes vanishingly small. All periodic structures have that property, but this is not limited to periodic structures. Disordered point patterns with a vanishing integral range have received some attention in the context of theoretical physics and they are referred to as being hyperuniform (Torquato \& Stillinger, 2003; Zachary \& Torquato, 2009). We shall illustrate this concept in the discussion section.

In terms of scattering, if the SAS pattern $I(q)$ scales like $q^{\nu}$ for small values of $q$, where $\nu$ is a constant, it results from Eq. (14) that the heterogeneity behaves like

$$
\sigma^{2}\{\Pi\} \sim 1 / L^{3+\nu}
$$

for large values of $L$. Any positive value of $\nu$ is therefore characteristic of a structure with vanishing integral range.

\subsection{Interpretation of $\sigma^{2}\{\Pi\}$ in terms of size distributions}

We have shown in the last two sections that the small-probe and large-probe limits of the scale-dependent heterogeneity $\sigma^{2}\{\Pi\}$ provide two different characteristic sizes of the underlying system, namely: the specific surface area and the integral range. The former and the latter are local and global characteristics of the structure, respectively. We now show how the $\sigma^{2}\{\Pi\}$ curves for intermediate probe sizes can be analysed in terms of size distributions.

It has to be stressed that the very concept of size is defined unambiguously only for geometrically simple structures. For example, a diameter can be used only when the structure can be idealised in terms of objects (particles or pores) that are almost spherical. In the case of complex structures like Figure 4, which cannot even be decomposed into isolated solid objects or pores there is no unique definition of size (see e.g. Serra (1982)). We propose here to define the size of an object, however complex, as the 
size $L$ of the probe $\Pi$ that is needed to filter that object out of the structure. With this definition, the estimated size of a structure depends on the particular type of probe chosen - spherical, Gaussian, or other - but this is merely a matter of definition.

To illustrate the relation between the scale-dependent heterogeneity $\sigma^{2}\{\Pi\}$ and the size, consider again the hierarchical structure in Figure 5a. In order to formalise the analysis, we shall denote the characteristics of the small and large scales with superscripts $(s)$ and $(l)$, respectively. Moreover, we still refer to the white phase as the solid $S$ and to the black phase as the pore $P$. The structure in Figure 5a comprises large solid regions that span across the entire image. The corresponding volume fraction at that scale is $\phi_{S}^{(l)} \simeq 0.5$. These large regions, however, are not homogeneous on a small scale because they are made up of finer structures. The volume fraction of the solid $S$ within these larger regions is $\phi_{S}^{(s)} \simeq 0.7$. The overall volume fraction of the solid in the entire structure is therefore $\phi_{S}=\phi_{S}^{(l)} \phi_{S}^{(s)} \simeq 0.35$. We now analyze in detail the geometrical characteristics of this hierarchical structure that contribute to the $\sigma^{2}\{\Pi\}$ curve, when the size $L$ of the probe is progressively increased. Here again, we discuss this in terms of the filtered density map $\rho_{\pi}(\mathbf{x})$ defined in Eq. (2).

When the probe is much smaller than the smallest objects in the structure, the effect of the filtering is only to blur the interfaces as in Figure $5 \mathrm{~b} 1$ for the spherical probe and b2 for the Gaussian probe. This is the range of $L$ where Eq. (25) applies, with $A_{P S}$ being the total surface area resulting from both the small-scale and large-scale structures. With larger probes, however, the entire small-scale structure is blurred out by the filtering operation, which is particularly clear in the case of the Gaussian probe in Figure 5b2. In that range of $L$ the small-scale structure is irrelevant. However, we expect Eq. (25) to remain valid but the relevant surface area is that of the largescale structure $A_{P S}^{(l)}$ only. Moreover, the relevant invariant $Q$ is that of the large-scale 
structure too, namely

$$
Q^{(l)}=\left[\left(\rho_{S}-\rho_{P}\right) \phi_{S}^{(s)}\right]^{2} \phi_{S}^{(l)}\left(1-\phi_{S}^{(l)}\right)
$$

This expression results from the classical form in the right-hand-side of Eq. (1), with the contrast term evaluated between the average density of the solid (after averaging out the small-scale structure) $\rho_{P}\left[1-\phi_{S}^{(s)}\right]+\rho_{S} \phi_{S}^{(s)}$ and $\rho_{P}$. Because the invariant of the entire structure can be written explicitly in terms of the small-scale and large-scale structures as

$$
Q=\left[\rho_{S}-\rho_{P}\right]^{2} \phi_{S}^{(l)} \phi_{S}^{(s)}\left(1-\phi_{S}^{(l)} \phi_{S}^{(s)}\right)
$$

the relevant expression of $\sigma^{2}\{\Pi\}$ for intermediate probe sizes is finally

$$
\frac{\sigma^{2}\{\Pi\}}{Q} \simeq \frac{\phi_{S}^{(s)}-\phi_{S}}{1-\phi_{S}}-\frac{\alpha_{\pi}^{(1)}}{4} \frac{\left[\phi_{S}^{(s)}\right]^{2}}{\phi_{S}\left(1-\phi_{S}\right)} A_{P S}^{(l)} L+\ldots
$$

For obtaining this expression we have used the fact that the volume fraction of the solid can be written as $\phi_{S}=\phi_{S}^{(s)} \phi_{S}^{(l)}$. The blue lines in Figure 6 correspond to Eq. (39), with the exact values of $\phi_{S}^{(s)}, \phi_{S}$ and $A_{P S}^{(l)}$ of the model in Figure 4 , and the values of $\alpha_{\pi}^{(1)}$ of the relevant probe. For all the probes, the line corresponding to Eq. (39) is found to be tangent to the heterogeneity curves $\sigma^{2}\{\Pi\}$ as it should.

It has also to be noted that the validity of Eq. (39) is not limited to two-scale structures, provided one understands the superscripts $(s)$ and $(l)$ as referring to whatever structure is smaller or larger than the considered probe size $L$. If Eq. (39) is understood as the tangent of the $\sigma^{2}\{\Pi\}$ curve, the quantity $\phi_{S}^{(s)}$ is related to the pore size distribution because only the pores smaller than $L$ are blurred out in $\rho_{\pi}$ and contribute to $\phi_{S}^{(s)}$. Similarly, $A_{P S}^{(l)}$ is to be understood as the surface area of the structures larger than $L$, which are the only ones to be still present in $\rho_{\pi}$ for probes of that size.

To make the relation to size distribution explicit, we define $F_{V}(L)$ as the cumulative pore-volume distribution. In other words, $\phi_{P} \times F_{V}(L)$ is the volume fraction of all pores

IUCr macros version 2.1.6: 2014/10/01 
smaller than $L$. Because $\phi_{S}+\phi_{P}=1$, this quantity is related to $\phi_{S}^{(s)}$ via

$$
\phi_{S}^{(s)}=1-\left(1-\phi_{S}\right) F_{V}(L)
$$

With that relation in mind, the first term on right-hand side of Eq. (39) is equal to $1-F_{V}(L)$. This means that the pore-size distribution can be obtained from the intercept of the tangent to the scale-dependent heterogeneity $\sigma^{2}\{\Pi\}$. The slope of the tangent is proportional to distribution of the surface area as a function of pore size. We define $F_{S}(L)$ as the cumulative surface distribution as

$$
A_{P S}^{(l)}=A_{P S}\left(1-F_{S}(L)\right)
$$

where $A_{P S}^{(l)}$ is calculated from the slope of Eq. (39), knowing the corresponding value of $\phi_{S}^{(s)}$ determined from the intercept.

The volume and surface distributions obtained in this way depend naturally on the type of probe chosen for the analysis. This reflects the fact that there is no unique definition of size for geometrically complex structures. The size distributions obtained from the SAXS pattern in Figure 4 using either a spherical or a Gaussian probe are shown in Figure 7a and 7b. The distributions exhibit a two-step increase. A first family of small pores - with $L \leq 20 \AA$ (Gaussian) and $L \leq 30 \AA$ (spheres) - accounts for about $\phi_{P}=0.30$ and $A_{P S}=0.02 \AA^{-1}$ for both probes. These values coincide reasonably with the known volume fraction and surface area of the micropores in model of Figure 4. It is also not surprising that for the same structure, the size $L$ defined with a Gaussian probe is smaller than with a spherical probe. This originates in the fact that the spherical probe is compact while the Gaussian has a tail extending far beyond the standard deviation $L$. The horizontal shifting of the distributions in Figure 7 merely results from a different definition of the size. The important observation is that the shape of the curves, i.e. the relative contribution of the micro- and meso-pores to the surface area and to the volume, is independent of the probe.

IUCr macros version 2.1.6: 2014/10/01 

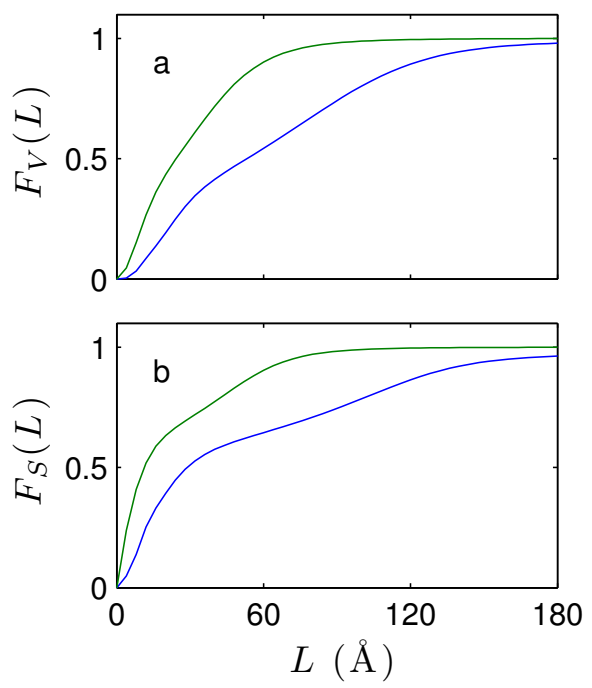

Fig. 7. Size distributions derived from the scale-dependent heterogeneity via Eq. (39). Cumulative distributions of the volume fraction (a) and of the specific surface area (b) against probes size $L$ in the case of a spherical (blue line) and Gaussian probes (green line).

Finally, it has to be stressed that the $q$-spherical probe cannot be used to determine size distributions. It was implicit in the discussion so far that increasing the size $L$ of the probe results in smoother values of the filtered density map $\rho_{\pi}(\mathbf{x})$. This is not necessarily the case with the $q$-sphere because its real-space structure contains oscillating positive-and-negative features (see Figure 6c). As a consequence, the values of $\rho_{\pi}$ are not necessarily bounded by $\rho_{P}$ and $\rho_{S}$. This is notably the case in Figure 5-b3, and it is responsible for the presence of inflection points in the heterogeneity curve $\sigma^{2}\{\Pi\}$ in Figure $7 \mathrm{c}$. Using blindly that curve to estimate $F_{S}(L)$ and $F_{V}(L)$ would lead to non-monotonic functions, which cannot be interpreted as cumulative distributions.

\section{Discussion}

We have derived general mathematical results concerning the structural significance of the scale-dependent heterogeneity $\sigma^{2}\{\Pi\}$, and we have illustrated them on the parIUCr macros version 2.1.6: 2014/10/01 
ticular case of a mathematical model of porous material comprising two families of pores (Figure 4). A variety of different scale-dependent heterogeneity curves can be obtained from scattering data depending on the particular type of probe function $\Pi$ that is used. However, we have shown that all these curves can be analysed consistently in terms of specific surface areas and integral ranges provided the relevant characteristics of the probe are taken into account (see Table 1). Therefore, the choice of any particular probe for analysing scattering data in terms of scale-dependent heterogeneity is merely a matter of convenience. In that respect the classical correlation function - corresponding to a probe $\Pi$ consisting of two points only- is just one among many mathematically equivalent functions. And it is not a particularly convenient one from the point of view of data analysis.

The most convenient probe in many respects is the one we referred to as a $q$-sphere, the real-space structure of which is given by Eq. (27). First, the evaluation of the corresponding $\sigma^{2}\{\Pi\}$ curve is straightforward via Eq. (28). This can be calculated on a spreadsheet and it does not require any extrapolation of the scattering data outside of the experimentally measured angular. Second, because the coefficient $\alpha_{\pi}^{(2)}$ of the $q$-sphere is equal to zero (Table 1 ) the $\sigma^{2}\{\Pi\}$ curves exhibit sharp linear regions, the slopes and intercepts of which can be determined unambiguously. The only situation we encountered in which the $q$-sphere was not suitable is the determination of size distributions, which requires a probe function that is compact is real-space, such as the sphere. In the present discussion section, we show how the concept of scale-dependent heterogeneity can be used to analyse experimental small-angle scattering data. We discuss here three different nanostructured materials, the SAXS data of which are plotted in Figure 8.

IUCr macros version 2.1.6: 2014/10/01 

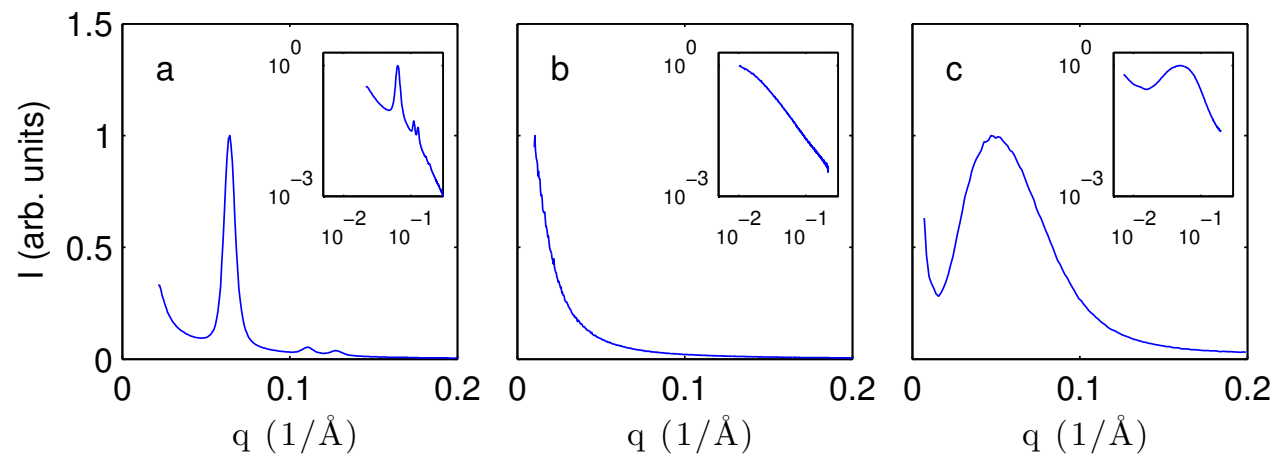

Fig. 8. Three qualitatively different SAXS patterns used to illustrate the usefulness of scale-dependent heterogeneity for practical data analysis. (a) micro- and mesoporous silica SBA-15, (b) silica alcogel obtained from the hydrolysis and condensation of TEOS, and (c) low-density semicrystalline polyethylene sample. The insets display the same data on double logarithmic scales.

The first material is so-called SBA-15 ordered micro- and meso-porous silica, synthesised by liquid-crystal templating (Zhao et al., 1998). The structure of that material is sketched in Figure 9: it consists in an hexagonal array of cylindrical mesopores having approximate diameter of $2 R=8 \mathrm{~nm}$, with the silica between the mesopores containing micropores (i.e. with sizes smaller than about $2 \mathrm{~nm}$ ). Nitrogen adsorption and desorption was measured on the very sample shown in Figure 8a (Gommes et al., 2015). The BET specific surface area of this material is $S_{B E T}=775 \mathrm{~m}^{2} / \mathrm{g}$ and the total pore volume is $V_{p}=0.79 \mathrm{~cm}^{3} / \mathrm{g}$. Using the value of the pore volume from nitrogen adsorption, and assuming a specific mass $\rho_{s}=2.2 \mathrm{~g} / \mathrm{cm}^{3}$ for the solid phase of that material, the overall solid fraction of SBA-15 is calculated as

$$
\phi_{S}=\frac{1}{1+\rho_{s} V_{p}} \simeq 0.37
$$

This numerical value is used hereafter for the SAXS data analysis. 


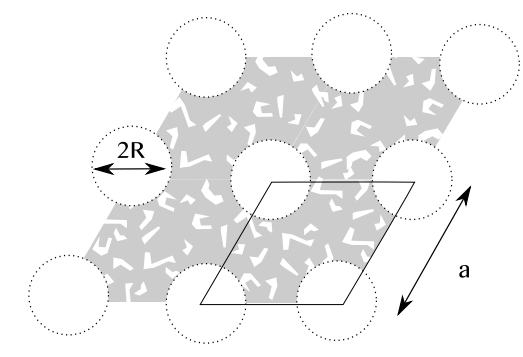

Fig. 9. Sketch of the structure of SBA-15 porous silica, exhibiting a hexagonal array (with lattice parameter $a$ ) of cylindrical mesopores with radius $R$. The solid line highlights the unit cell. The space between mesopores contains disordered micropores.

The second material (Figure 8b) is a silica alcogel synthesized from the hydrolysis and polycondensation of tetraetyoxysilane (TEOS) in ethanol (Gommes et al., 2007). Because the word silica gel is sometimes abusively used to describe the porous material obtained after desiccating such a gel, it is useful to stress that the material investigated here is still soaked in the solvent in which it was synthesised. As a consequence, the scattering pattern of the material does not exhibit Porod scattering with exponent 4 . Instead, an asymptotic power-law scattering with an exponent close to 2 is observed, which is typical of gels. The solid fraction of the gel, assuming that the condensation reactions have proceeded to the point where the solid consists in dense $\mathrm{SiO}_{2}$, is estimated from the overall composition of the starting solution to be $\phi_{S} \simeq 0.15$.

Finally, the third and last material (Figure 8c) is a semicrystalline low-density polyethyene (LDPE) sample (Basiura, 2005). In that material, the SAXS intensity results from the electron density contrast between the nanometer-sized crystalline and molten regions. In order to keep the same notation as in the theoretical part of the paper, we refer hereafter to the crystalline and molten phases as $S$ and $P$, respectively. Using calorimetry, the crystallinity (i.e. the volume fraction of the crystalline phase) of that material is estimated to be $\phi_{S} \simeq 0.2$ (Basiura, 2005).

The scale-dependent heterogeneities $\sigma^{2}\{\Pi\}$ calculated from the SAXS patterns in IUCr macros version 2.1.6: 2014/10/01 
Figure 8 with $q$-spherical probes are plotted in Figure 10. The curves of the SBA-15 and LDPE samples display a clear linear portion close to $L=0$, from which the following values are obtained for the specific surface areas: $A_{P S}=403 \mathrm{~m}^{2} / \mathrm{cm}^{3}$ for SBA-15 and $A_{P S}=241 \mathrm{~m}^{2} / \mathrm{cm}^{3}$ for LDPE.
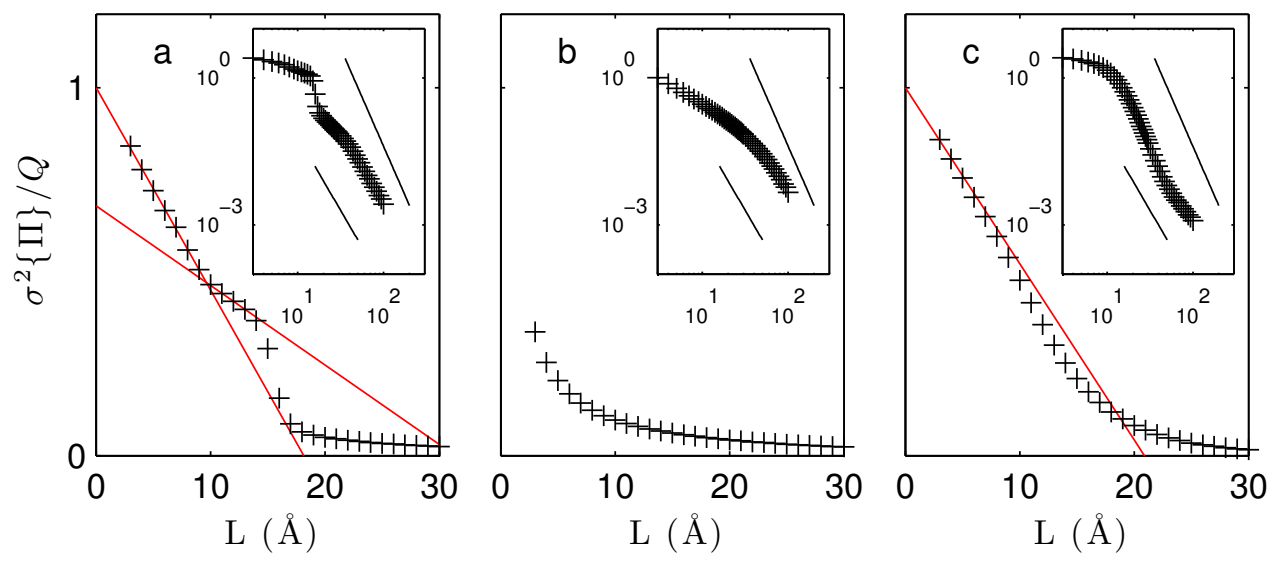

Fig. 10. Scale-dependent heterogeneity curves estimated from the scattering data in Figure 8 with a $q$-spherical probe, i.e. with eq. (28): (a) SBA-15, (b) TEOS and (c) LDPE. The solid red lines are fits of the linear portions of the plots, when applicable (see text). The insets display the same curves on double logarithmic scales. The two lines in each inset are power laws with exponents -3 and -4 .

An interesting feature of the $\sigma^{2}\{\Pi\}$ curves of SBA-15 is the presence of a second linear region for $L \simeq 10 \AA$, in much the same way as in Figure 6c. This results from the hierarchical structure of the material, which comprises both meso- and micropores (see Figure 9). Using Eq. (39) to analyse the slope and intercept of that second linear region, one obtains the values $\phi_{S}^{(s)}=0.79$ and $A_{P S}^{(l)}=253 \mathrm{~m}^{2} / \mathrm{cm}^{3}$. The first value is the solid fraction of the silica wall between neighbouring mesopores. That value compares reasonably with estimates obtained by a variety of techniques on the same type of material, which all point a wall porosity of the order of $30 \%$ (Hofmann et al., 2005; Gommes et al., 2009; Prass et al., 2009). Concerning the value of $A_{P S}^{(l)}$, it corresponds to the surface area of the structures that remain in the density map $\rho_{\pi}(\mathbf{x})$ after filtering with a $q$-spherical probe of size $L \simeq 10 \AA$. The relation between IUCr macros version 2.1.6: 2014/10/01 
$L$ and the actual size of the structures is probe-dependent. From Figure 3c, the actual width (diameter) of the $q$-spherical probe in real space is about $4 L$. In the particular case of SBA-15, it is therefore safe to assume that micropores (i.e. with width smaller than $20 \AA$ ) do not contribute to the linear region at $L \simeq 10 \AA$. The surface area $A_{P S}^{(l)}$ corresponds therefore to that of the mesopores only. In order to verify this, one can relate the mesopore surface area $A_{P S}^{(m e s o)}$ to the radius $R$ of the mesopores, using the geometrical model in Figure 9. This leads to

$$
A_{P S}^{(m e s o)}=\frac{2 \pi R}{a^{2} \sqrt{3} / 2}
$$

where the numerator is the perimeter of mesopore circular section and the denominator is the area of the hexagonal unit cell. The value of the lattice parameter $a$ is obtained from the position of the first peak in the scattering pattern via $q=4 \pi /(a \sqrt{3})$ (Förster et al., 2005), which leads to $a=113 \AA$ for the data in Figure 8a. Using that value of $a$ and $A_{P S}^{(l)}=252 \mathrm{~m}^{2} / \mathrm{cm}^{3}$ leads to the mesopore radius $R=45 \AA$. This value compares beautifully with the estimated radius from nitrogen adsorption of $40 \AA$ as mentioned earlier, as well as with the value $R=43 \AA$ obtained via a thorough modelling of the scattering pattern (Gommes et al., 2016).

In order to compare the total surface area $A_{P S}=403 \mathrm{~m}^{2} / \mathrm{cm}^{3}$ to the BET surface area measured from nitrogen adsorption, one has to convert it first to $\mathrm{m}^{2} / \mathrm{g}$. This requires dividing by the bulk density of the material $\phi_{S} \rho_{s}=0.81 \mathrm{~g} / \mathrm{cm}^{3}$. The corresponding value is $A_{P S}=497 \mathrm{~m}^{2} / \mathrm{g}$, which is significantly smaller than the $775 \mathrm{~m}^{2} / \mathrm{g}$ according to BET. The difference points to a significant molecular-scale roughness of the surface, which increases the BET area beyond its geometrical value (Gregg \& Sing, 1982).

It has to be stressed that despite the hierarchical structure of SBA-15, with both micro- and meso-pores, its SAXS pattern in Figure 8a does not exhibit a double Porod plateau that would enable one to discriminate simply the surface area of the IUCr macros version 2.1.6: 2014/10/01 
two types of pores (Ciccariello, 1988). The scattering pattern is dominated by the Bragg peaks resulting from the hexagonal periodicity of the mesopore structure at intermediate values of $q$. This makes a classical Porod analysis difficult. By contrast the two linear regions in the $\sigma^{2}\{\Pi\}$ plot are very clear in Figure 10a. It is because the present analysis focuses on the heterogeneity, rather than on the periodicity, that the hierarchical structure of the material is made apparent.
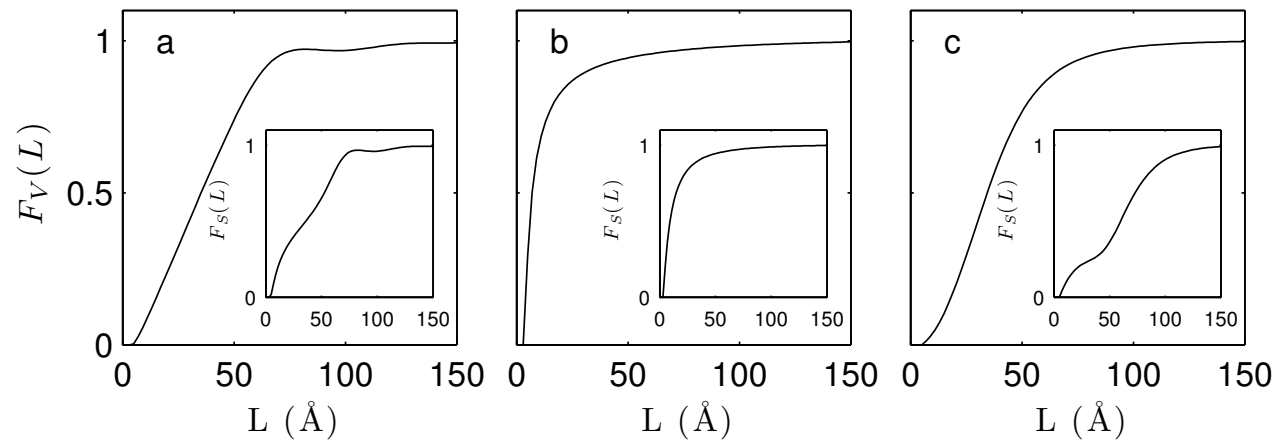

Fig. 11. Size distributions of the SBA-15, TEOS and LDPE samples derived from the scale-dependent heterogeneity via Eq. (39). Cumulative distributions of the volume fraction (main graphs) and of the specific surface area (insets) against probe size $L$. A spherical probe was used for the calculations.

The main structural characteristics of the SBA-15 sample are also visible in the size distributions plotted in Figure 11a. Those distributions were obtained as in Section 3.3, based on the scale-dependent heterogeneity curve with a spherical probe. The volume and surface distributions $F_{V}(L)$ and $F_{S}(L)$ exhibit a riser around $L=50 \AA$, which corresponds to the size of the mesopores. The riser is better defined in the case of the surface distribution (inset), which exhibits a two-step increase. The first one saturating around $L \simeq 20 \AA$ shows that half of the surface area is due to micropores in SBA-15.

In the case of the gel sample (TEOS, Figure 10b) no linear region can be detected in the heterogeneity curve: the curve becomes steeper and steeper when $L$ becomes vanishingly small. Mathematically, this results from the asymptotic scattering of that IUCr macros version 2.1.6: 2014/10/01 
sample following a power law of the type $I \sim q^{-2}$. Because this decrease is slower than $q^{-3}$, the integral in Eq. (14) does not converge for infinitely small probes. The traditional way to describe this type of scattering in the SAXS literature is in terms of a mass fractal, i.e. with fractal dimension smaller than 3 (Schmidt, 1991). The fact that the heterogeneity diverges for small $L$ 's, points at the appearance of new structures each time the observation scale is reduced. In a sense, this is the very essence of a mass fractal. The overall fractal structure of the TEOS sample is also visible in the size distributions $F_{V}(L)$ and $F_{S}(L)$ (Figure 11b), which are extremely steep for small values of $L$.

Finally, in the case of the semicrystalline LDPE (Figure 10c) the surface area $A_{P S}=$ $241 \mathrm{~m}^{2} / \mathrm{cm}^{3}$ can be converted to thicknesses of the crystalline and amorphous domains. Assuming a lamellar structure, as usual for this type of material, leads to the average thicknesses $l_{S}=2 \phi_{S} / A_{P S} \simeq 17 \AA$ for the crystalline domains and $l_{P}=2 \phi_{P} / A_{P S} \simeq 66$ $\AA$ for the amorphous regions. The two-step increase of $F_{S}(L)$ (inset of Figure 11c) should not be interpreted here in terms of a hierarchical structure. In the case of LDPE the first riser around $L \simeq 20 \AA$ is a consequence of the flattening of the Porod scattering for large values of $q$ (inset of Figure 8c). This flattening results from the scattering of the polymer melt, and not from the substructure of the crystals. The size distributions should therefore be interpreted carefully.

The most striking and interesting characteristic of the heterogeneity curve of the LDPE sample is not apparent for small probes but for large probes (see inset of Figure 10c). Unlike SBA-15 and TEOS for which $\sigma^{2}\{\Pi\}$ scales like $L^{-3}$, the scaling is proportional to $L^{-4}$ for LDPE. As mentioned in Section 3.2, this type of scaling is characteristic of structures with a vanishing integral range, namely

$$
\int_{0}^{\infty} \gamma(r) 4 \pi r^{2} \mathrm{~d} r=0
$$

In reciprocal space, this statement is equivalent to stating that the scattered intensity IUCr macros version 2.1.6: 2014/10/01 
$I(q)$ extrapolates to 0 for small values of $q$. This is indeed the case for angular range from $q \simeq 0.01 \AA^{-1}$ to $q \simeq 0.5 \AA^{-1}$, corresponding to left side of the broad scattering maximum in Figure 8c. At very small angles (for $q<0.01 \AA^{-1}$ ) the scattered intensity increases again, which leads to a flattening of the $\sigma^{2}\{\Pi\}$ curve in the inset of Figure 10c around $L=100 \AA$.
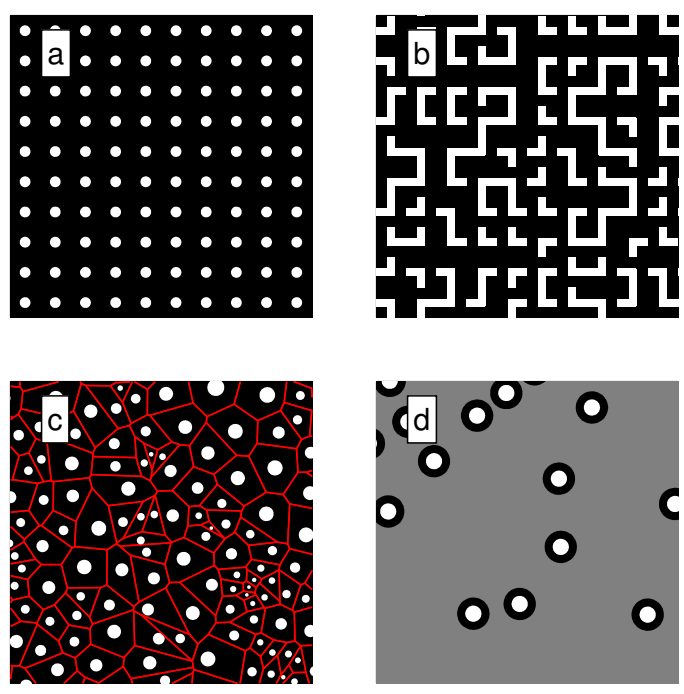

Fig. 12. Examples of hyperuniform structures, that is with vanishing integral range $\left(\mu_{0}^{\gamma}=0\right)$ : (a) periodic structure, (b) crystalline structure with random orientation of the motif, (c) disordered system with strongly correlated sizes (see text), and (d) diffusion-limited systems in which each high-density region is surrounded by a depleted region. In (c) the red lines are the limit of the Poisson-Voronoi cells from which the structure is built; in (d) the grey scale symbolises the electron density.

In order to discuss the structural meaning of a vanishing integral range, four qualitatively different structures having that property are displayed in Figure 12. Fundamentally, having $\mu_{0}^{\gamma}=0$ means that when a large probe (size $L$ ) is moved systematically through the structure, the variance of the total number of electrons inside the probe does not scale proportionally its volume $L^{3}$ (like a Poisson process would), but to its outer surface area $L^{2}$ (Torquato \& Stillinger, 2003). This is notably expected in the case of a periodic structure such as Figure 12a, for which all the unit cells are identical. In that case, the reason for the fluctuations of the number of electrons is that some IUCr macros version 2.1.6: 2014/10/01 
unit cells are intersected by the outer surface of the probe, so that a random fraction of their electrons are inside. The number of intersected unit cells scales like the area of the intersecting surface, i.e. as $L^{2}$. The exponents $L^{-3}$ or $L^{-4}$ shown in the insets of Figure 10 result from converting the total number of electrons to an electron density. The same reasoning applies to structures obtained by repeating a motif with random orientation, as shown in Figure 12b.

The first two examples in Figure 12 show that the only thing that matters to ensure $\mu_{0}^{\gamma}=0$ is the density within each cell of the lattice. Building on that observation, it is possible to construct disordered (non-periodic) structures with a vanishing integral range. Consider for example the structure in Figure 12c. It was obtained in two steps by performing first a Poisson-Voronoi tessellation (Lantuéjoul, 2002), whereby the plane is decomposed into a set of convex regions with finite areas (red cells). As a second step each Voronoi cell is occupied by a particle, the size of which is chosen in such a way that it fills exactly $10 \%$ of cell. This two-step procedure leads to a disordered polydisperse system with strong correlations between the size of particles: isolated particles are large and clustered particles are small. With such a structure, the fluctuation of the number of electrons in a random probe volume results from the Voronoi cells that are intersected by the surface of the probe. The cells that lie entirely within the probe do not contribute to the fluctuations. Although the structure in Figure 12c is disordered, its integral range is zero.

It is interesting to note that the geometrical construction based on Poisson-Voronoi cells is physically similar to the nucleation-and-growth scenario of phase separation. In that context, nuclei are formed randomly in space and they subsequently grow when molecules in their neighbourhood attach to them. This process naturally leads to disordered structures with strong correlations between the size of the particles and their spatial distribution. Clustered particles are necessarily small because the

IUCr macros version 2.1.6: 2014/10/01 
molecules initially in their neighbourhood had to be shared among many particles. For the same reason, isolated particles are comparatively larger. A similar mechanisms is expected in the case of polymer crystallisation. In that context, crystalline regions grow by expelling entangled polymer chains that necessarily become concentrated in the surrounding region, which eventually prevents further crystal growth. It is beyond the scope of the present paper to discuss thoroughly these questions, but this would be a plausible explanation for the vanishing integral range of the structure of LPDE. Models similar to Figure 12b are expected to be relevant for a variety of materials. A classical example is the one-dimensional so-called migration model discussed by Serra (1982), which was proposed independently by Fratzl et al. (2005) in the context of SAXS analysis of mineral platelets in bones.

Yet another type of structure with $\mu_{0}^{\gamma}=0$ that can be encountered in materials science corresponds to the case of diffusion-limited growth, whereby a growing phase is surrounded by a boundary layer that is depleted of the molecules that it is made of. In Figure 12c, the grey level symbolises the concentration, or the electron density. Because the number of electrons is conserved during the growth, the total number of electrons in a given region of space is unchanged if an initially homogeneous system is replaced by a denser phase surrounded by a depleted layer. Therefore it is only the phases and boundary layers that are intersected by the probe's outer surface that contribute to the fluctuations of the number of electrons. This type of situation is accordingly expected to yield scattering patterns that extrapolate to 0 for small values of $q$, which is indeed observed experimentally for metal nanoparticles growing inside a glass matrix (Craievich et al., 2002).

\section{Conclusions}

The structural information contained in small-angle scattering patterns can be conIUCr macros version 2.1.6: 2014/10/01 
veniently analysed in terms of scale-dependent heterogeneity. The general procedure that we proposed to quantify heterogeneity consists in estimating the average density of the material within a given probe volume, and analysing the density fluctuations when the probe is moved systematically through the material. The correlation function $\gamma(r)$ is a particular case corresponding to a probe consisting of only two points at distance $r$ from one another, but the procedure is quite general. We have considered spherical probes, Gaussian probes, as well as probes defined as spheres in reciprocal space. In all cases, it is the linear size of the probe that sets the scale of the analysis. All the corresponding measures of heterogeneity are mathematically equivalent because they contain the same type of geometrical information. For the purpose of scattering data analysis, using one type of probe instead of another is merely a matter of convenience.

Although the correlation function is conceptually the simplest measure of heterogeneity, from which all the others can be easily derived, it is not necessarily the most convenient from the point of view of scattering data analysis. An extremely convenient probe in that respect is the sphere in reciprocal space. The corresponding scale-dependent heterogeneity can be calculated easily from experimental scattering patterns, without invoking indirect Fourier transforms or extrapolating the data outside of the measured angular range. Despite the simplicity of the analysis, we have shown how it enables one to determine surface areas and volume fractions of materials with complex and hierarchical structures.

From an almost philosophical perspective, it is interesting to note the similarity between the structural analysis of scale-dependent heterogeneity curves and the general procedure of morphological analysis advocated by Serra (1982). In the spirit of mathematical morphology any structure can be characterised by measuring how it is affected by the application of a given filter. It is therefore the filter that determines the

IUCr macros version 2.1.6: 2014/10/01 
nature of the measurement. In the context of image analysis non-linear filters are generally applied, such as morphological opening or closing, and volumes are measured. The transformation of the density map $\rho(\mathbf{x})$ into $\rho_{\pi}(\mathbf{x})$ via Eq. (2) is a filter too. The difference with mathematical morphology is that only linear filters are possible for scattering data analysis because their effects have to be calculated in reciprocal space. This parallel sheds an interesting light on the so-called phase problem of crystallography, i.e. on the question of the missing structural information when only the scattering pattern of a structure is known (Gommes et al., 2012b). From a practical point of view, we have shown that linear filters were sufficient to analyse the surface areas and densities of hierarchical micro- and meso-porous materials. Moreover, the different perspective offered by the concept of heterogeneity and fluctuations enabled us to analyse structures in a new light, using for instance the unifying concept of integral range and hyperuniformity.

The present contribution raises many questions and opens the way to a variety of further developments. For example, it is in principle possible to design probe volumes such that the leading term in $\sigma^{2}\{\Pi\}$ for small probes would be related to the curvature of the interfaces rather than to their area. Mathematically speaking, this would require a probe with $\mu_{1}^{\Omega}=0$. Can that be used to design data analysis procedures to estimate non-trivial structural characteristics? All probes considered here are equivalent to low-pass filters; would there be an interest in considering high-pass filters as well? This would be equivalent to considering probes with $\int \Pi(\mathbf{x}) \mathrm{d} V_{x}=0$. As another example, we considered here only the one-way calculation from the scattering pattern to the heterogeneity, which proved mathematically simple for any type of probe. Can we design a family of probes such that the reverse transformation, from the heterogeneity to the scattering pattern, would be simple too? If that were the case, we could imagine novel numerical procedures to reconstruct structures that match any

IUCr macros version 2.1.6: 2014/10/01 
given scattering dataset. Would there be an interest in inferring the entire distribution of average densities $\rho_{\pi}$ starting from the measured average and variance, say by maximum-entropy methods? Could structural information be obtained in that way? Is there a relation between the heterogeneity calculated with a rod-like probe and the concept of chord length distribution? Etc. We hope to investigate some of these questions in future work.

The author is grateful to the Funds for Scientific Research (F.R.S.-FNRS, Belgium) for a Research Associate position, to Prof. Bart Goderis (University of Leuven) and Dr. Monika Basiura (University of Bielsko-Biała) for providing the SAXS data of the polyethylene sample and for fruitful discussions, as well as to Prof. Dominique Jeulin (École des Mines de Paris) for bringing the field of geostatistics to his attention. The author is also grateful to Dr. Brian Pauw (Bundesanstalt für Materialforschung) for openly reviewing the present manuscript, and for his helpful suggestions.

\section{References}

Basiura, M. (2005). Non Classical Polyethylene Structures Explored by SAXS. Phd thesis. KULeuven, Belgium.

Chandler, D. (1987). Introduction to Modern Statistical Mechanics. New York: Oxford University Press.

Chilès, J.-P. \& Delfiner, P. (1999). Geostatistics: Modeling Spatial Uncertainty. New York: Wiley.

Ciccariello, S. (1988). J. Appl. Cryst. 21, 117-128.

Ciccariello, S. (1995). J. Math. Phys. 36(1), 219-246.

Ciccariello, S., Cocco, G., Benedetti, A. \& Enzo, S. (1981). Phys. Rev. B, 23(12), 6474-6485.

Craievich, A., Kellermann, G., Barbosa, L. \& Alves, O. (2002). Phys. Rev. Lett. 89(23), $235503 / 1-4$.

Debye, P., Anderson, H. R. \& Brumberger, H. (1957). J. Appl. Phys. 28(6), 679-683.

Förster, S., Timmann, A., Konrad, M., Schellbach, C., Meyer, A., Funari, S., Mulvaney, P. \& Knott, R. (2005). J. Phys. Chem. B, 109(4), 1347-1360.

Fratzl, P., Gupta, H. S., Paris, O., Valenta, A., Roschger, P. \& Klaushofer, K. (2005). Progr. Colloid Polym. Sci. 130, 33-39.

Glatter, O. (1977). J. Appl. Cryst. 10(5), 415-421.

Glatter, O. \& Kratky, O. (1982). Small Angle X-ray Scattering. New York: Academic Press.

Gommes, C. J., Friedrich, H., Wolters, M., de Jongh, P. E. \& de Jong, K. P. (2009). Chem. Mater. 21, 1311-1317.

Gommes, C. J., Goderis, B., Pirard, J.-P. \& Blacher, S. (2007). J. Non-Cryst. Solids, 353, $2495-2499$.

Gommes, C. J., Jiao, Y. \& Torquato, S. (2012a). Phys. Rev. Lett. 108(8), 080601.

IUCr macros version 2.1.6: 2014/10/01 
Gommes, C. J., Jiao, Y. \& Torquato, S. (2012b). Phys. Rev. E, 85, 051140.

Gommes, C. J., Prieto, G. \& De Jongh, P. E. (2016). J. Phys. Chem C, 120, 1400-1506.

Gommes, C. J., Prieto, G., Zecevic, J., Vanhalle, M., Goderis, B., de Jong, K. P. \& de Jongh, P. E. (2015). Angew. Chem. Int. Ed. 54(40), 11804-11808.

Goodisman, J. \& Brumberger, H. (1971). J. Appl. Cryst. 4, 347-351.

Gregg, S. J. \& Sing, K. S. W. (1982). Adsorption, Surface Area and Porosity. London: Academic Press.

Guinier, A. \& Fournet, G. (1955). Small Angle Scattering of X-rays. New York: John Wiley.

Gupta, H., Seto, J., Wagermaier, W., Zaslansky, P., Boesecke, P. \& Fratzl, P. (2006). Proc. Nat. Acad. Sci. USA, 103(47), 17741-17746.

Hofmann, T., Wallacher, D., Huber, P., Birringer, R., Knorr, K., Schreiber, A. \& Findenegg, G. (2005). Phys. Rev. B, 72(6).

Kanit, T., Forest, S., Galliet, I., Mounoury, V. \& Jeulin, D. (2003). Int. J. Solids Struc. 40(13-14), 3647-3679.

Kirste, R. \& Porod, G. (1962). Kolloid-Zeitschrift \& Zeitschrift fr Polymere, 184(1), 1-7.

Lantuejoul, C. (1991). J. Microsc. 161(3), 387-403.

Lantuéjoul, C. (2002). Geostatistical Simulations. Springer.

Lu, B. L. \& Torquato, S. (1990). J. Chem. Phys. 93(5), 3452-3459.

Matheron, G. (1971). In Les Cahiers du Centre de Morphologie Mathématique de Fontainebleau, vol. 5, pp. 1-208. École Nationale Supérieure des Mines de Paris.

URL: http://cg.ensmp.fr/bibliotheque/public/

Pedersen, J. S. (1997). Adv. Coll. Interf. Sci. 70, 171-210.

Pietronero, L., Gabrielli, A. \& Labini, F. S. (2002). Physica A, 306, 395-401.

Porod, G. (1948). Acta Phys. Austr. 3, 255-292.

Prass, J., Müter, D., Fratzl, P. \& Paris, O. (2009). Appl. Phys. Lett. 95(8).

Ruland, W. (1971). J. Appl. Cryst. 4, 70-73.

Schmidt, P. W. (1991). J. Appl. Cryst. 24(pt 5), 414-435.

Serra, J. (1982). Image Analysis and Mathematical Morphology, vol. 1. London: Academic Press.

Sivia, D. S. (2011). Elementary Scattering Theory. Oxford: Oxford University Press.

Soille, P. (1999). Morphological Image Analysis, principles and applications. Berlin, Heidelberg: Springer.

Svergun, D. (1999). Biophys. J. 76(6), 2879-2886.

Tchoubar, D. \& Méring, J. (1969). J. Appl. Cryst. 2, 128-138.

Torquato, S. (2000). Random Heterogeneous Materials. New York: Springer.

Torquato, S. \& Stillinger, F. H. (2003). Phys. Rev. E, 68, 411131-4111325.

Zachary, C. E. \& Torquato, S. (2009). J. Stat. Mech. $2009(12)$, P12015.

Zhao, D., Feng, J., Huo, Q., Melosh, N., Fredrickson, G., Chmelka, B. \& Stucky, G. (1998). Science, $\mathbf{2 7 9}(5350), 548-552$.

\section{Synopsis}

We define a quantitative measure for the scale-dependent heterogeneity of a structure, which can be calculated from small-angle scattering data. That measure generalises the notion of correlation function, and it leads to novel data analysis procedures.

IUCr macros version 2.1.6: 2014/10/01 